\title{
On the novel existence results of solutions for a class of fractional boundary value problems on the cyclohexane graph
}

\author{
Wajahat Ali', Ali Turab2 ${ }^{2 *}$ (i) and Juan J. Nieto ${ }^{3 *}$
}

*Correspondence:

taurusnoor@yahoo.com;

juanjose.nieto.roig@usc.es

${ }^{2}$ Department of Mathematics and

Statistics, Faculty of Science and

Technology, Thammasat University

Rangsit Center, Pathum Thani

12120, Thailand

${ }^{3}$ Instituto de Matemáticas,

Universidade de Santiago de

Compostela, 15782 Santiago de

Compostela, Spain

Full list of author information is

available at the end of the article

\section{Springer}

\begin{abstract}
A branch of mathematical science known as chemical graph theory investigates the implications of connectedness in chemical networks. A few researchers have looked at the solutions of fractional differential equations using the concept of star graphs. Their decision to use star graphs was based on the assumption that their method requires a common point linked to other nodes but not to each other. Our goal is to broaden the scope of the method by defining the idea of a cyclohexane graph, which is a cycloalkane with the molecular formula $\mathrm{C}_{6} \mathrm{H}_{12}$ and CAS number 110-82-7. It consists of a ring of six carbon atoms, each bonded with two hydrogen atoms above and below the plane with multiple junction nodes. This article examines the existence of fractional boundary value problem' solutions on such graphs in the sense of the Caputo fractional derivative by using the well-known fixed point theorems. In addition, an example is given to support our key findings.
\end{abstract}

Keywords: Cyclohexane graph; Fractional calculus; Fixed points

\section{Introduction}

In fractional differential equations, there has been a lot of theoretical and practical development in recent years (for detail, see [1-4]). Most articles and books on fractional calculus in the setting of special functions concentrate on the solvability of linear fractional differential equations [5-13]. Several new papers dealing with nonlinear fractional differential equations and their solutions using methods such as the stability analysis, LeraySchauder theorem, and fixed point analysis have recently been published [14-19].

Chemical graph theory is one of the fields of mathematics that examines the consequences of a chemical network's connectivity. A chemical graph may represent any actual or abstract chemical system (i.e., molecular transformations in a chemical reaction). Chemical graph theory, in other words, is focused on every element of graph theory's application to chemistry. Furthermore, in chemical graph theory, the word "chemical" is used to highlight that, unlike graph theory, one may depend on scientific observation of many ideas and theorems rather than rigorous mathematical proofs, which is a crucial difference.

(c) The Author(s) 2022. This article is licensed under a Creative Commons Attribution 4.0 International License, which permits use sharing, adaptation, distribution and reproduction in any medium or format, as long as you give appropriate credit to the original author(s) and the source, provide a link to the Creative Commons licence, and indicate if changes were made. The images or other third party material in this article are included in the article's Creative Commons licence, unless indicated otherwise in a credit line to the material. If material is not included in the article's Creative Commons licence and your intended use is not permitted by statutory regulation or exceeds the permitted use, you will need to obtain permission directly from the copyright holder. To view a copy of this licence, visit http://creativecommons.org/licenses/by/4.0/. 
Figure 1 A picture of a star graph $\mathbb{G}^{\star}$

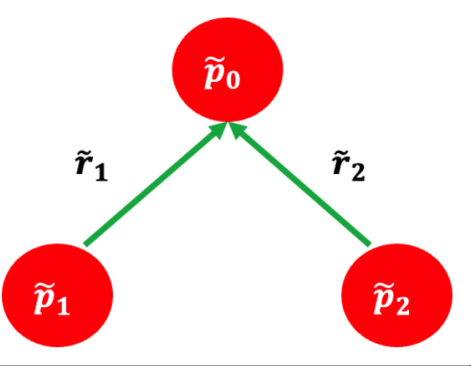

Lumer's research [20] was the first to look at differential equation theory applied to graphs. By altering specified local operators, he investigated extended equations of evolution on ramification spaces. In 1989, using a geometric network, Zavgorodnij [21] studied linear differential equations, with the existence of suggested boundary value problem solutions arranged at the interior vertices of the network. Gordeziani et al., on the other hand, used the double-sweep technique to find the numerical solutions for ordinary differential equations on graphs, which they found to be more efficient (see [22]).

However, just a few studies on fractional boundary value problems with graphs have shown the existence of solutions using specific fixed point techniques (see [23, 24]). The concept of a star graph was used by the authors of these works, namely $\mathcal{E}^{\star}=\mathcal{X}^{\star}\left(\mathcal{E}^{\star}\right) \cup$ $\mathcal{Y}^{\star}\left(\mathcal{E}^{\star}\right)$, where $\mathcal{X}^{\star}\left(\mathcal{E}^{\star}\right)=\left\{\tilde{p}_{0}, \tilde{p}_{1}, \tilde{p}_{2}\right\}$ and $\mathcal{Y}^{\star}\left(\mathcal{E}^{\star}\right)=\left\{\tilde{r}_{1}=\overrightarrow{\tilde{p}_{1}} \vec{p}_{0}, \tilde{r}_{2}=\overrightarrow{\tilde{p}_{2} \tilde{p}_{0}}\right\}$ are sets of vertices and edges, respectively, such that $\tilde{p}_{0}$ is the junction node and $\tilde{r}_{\ell}=\overrightarrow{\tilde{p}_{\ell} \tilde{p}_{0}}$ is the edge connecting nodes $\tilde{p}_{\ell}$ to $\tilde{p}_{0}$ having length $\tilde{\varrho}_{\ell}=\left|\overrightarrow{\tilde{p}_{\ell} \tilde{p}_{0}}\right|$ for $\ell=1,2$ (see Fig. 1).

After establishing a local coordinate system with the origin at the vertices $\tilde{r}_{1}$ and $\tilde{r}_{2}$, the coordinate $s \in\left(0, \tilde{\varrho}_{\ell}\right)$ is investigated on each edge $\tilde{r}_{\ell}=\overrightarrow{\tilde{p}_{\ell} \tilde{p}_{0}}$ (as seen in Fig. 1). Graef et al. [23] suggested the subsequent system of nonlinear fractional differential equations defined on each edge $\tilde{r}_{\ell}=\overrightarrow{\tilde{p}_{\ell} \tilde{p}_{0}}$ and applied the notable fixed point theorems to prove the existence of solutions of the following problem:

$$
\left\{\begin{array}{l}
-{ }^{\mathrm{RL}} \mathfrak{D}^{j} w_{\ell}(s)=h_{\ell}(s) L_{\ell}(s, y(s)) \quad\left(s \in\left(0, \tilde{\varrho}_{\ell}\right), \ell=1,2\right), \\
w_{1}(0)=w_{2}(0)=0, \quad w_{1}\left(\tilde{\varrho}_{1}\right)=w_{2}\left(\tilde{\varrho}_{2}\right), \quad{ }^{\mathrm{RL}} \mathfrak{D}^{k} w_{1}\left(\tilde{\varrho}_{1}\right)+{ }^{\mathrm{RL}} \mathfrak{D}^{k} w_{2}\left(\tilde{\varrho}_{2}\right)=0,
\end{array}\right.
$$

where $j \in(1,2], k \in(0, j), h_{\ell}:\left[0, \tilde{\varrho}_{\ell}\right] \rightarrow \mathbb{R}$ are continuous functions with $h_{\ell}(s) \neq 0$ on $\left[0, \tilde{\varrho}_{k}\right]$ and also $L_{\ell}:\left[0, \tilde{\varrho}_{\ell}\right] \times \mathbb{R} \rightarrow \mathbb{R}$ are continuous functions. Also, ${ }^{\mathrm{RL}} \mathfrak{D}^{j}$ and ${ }^{\mathrm{RL}} \mathfrak{D}^{k}$ represent Riemann-Liouville fractional derivatives of orders $j$ and $k$, respectively.

In [24], Mehandiratta et al. broaden the above work to $(u+1)$ vertices with

$$
\mathcal{X}^{\star}\left(\mathcal{E}^{\star}\right)=\left\{\tilde{p}_{0}, \tilde{p}_{1}, \ldots, \tilde{p}_{u}\right\} \quad \text { and } \quad \mathcal{Y}^{\star}\left(\mathcal{E}^{\star}\right)=\left\{\tilde{r}_{1}=\overrightarrow{\tilde{p}_{1} \tilde{p}_{0}}, \tilde{r}_{2}=\overrightarrow{\tilde{p}_{2} \tilde{p}_{0}}, \ldots, \tilde{r}_{u}=\overrightarrow{\tilde{p}_{u} \tilde{p}_{0}}\right\}
$$

where the length of each $\tilde{r}_{\ell}$ joining vertices $\tilde{p}_{\ell}$ to $\tilde{p}_{0}(\ell=1,2, \ldots, u)$ is $\tilde{\varrho}_{\ell}=\left|\overrightarrow{\tilde{p}_{\ell} \tilde{p}_{0}}\right|$. They investigated the solutions of the following problem:

$$
\left\{\begin{array}{lr}
\mathfrak{D}^{j} w_{\ell}(s)=\mathcal{H}_{\ell}\left(s, w_{\ell}(s), \mathfrak{D}^{k} w_{\ell}(s)\right) \quad\left(s \in\left(0, \tilde{\varrho}_{\ell}\right), \ell=1,2, \ldots, u\right), \\
w_{\ell}(0)=0, \quad w_{\ell}\left(\tilde{\varrho}_{\ell}\right) \neq w_{m}\left(\tilde{\varrho}_{m}\right) \quad(\ell \neq m), \sum_{\ell=1}^{u} w_{\ell}^{\prime}\left(\tilde{\varrho}_{\ell}\right)=0,
\end{array}\right.
$$


where $j \in(1,2], k \in(0, j-1], \mathcal{H}_{\ell}:\left[0, \tilde{\varrho}_{\ell}\right] \times \mathbb{R} \times \mathbb{R} \rightarrow \mathbb{R}$ are continuous functions and $\mathfrak{D}^{x}$ denotes the Caputo fractional derivative of order $\chi \in\{j, k\}$, and used transformations $\tilde{\theta}=$ $\frac{s}{\tilde{\varrho_{\ell}}} \in[0,1]$ and $\sigma(\tilde{\theta})=w(s)=w\left(\tilde{\varrho}_{\ell} \tilde{\theta}\right)$ for $s \in\left[0, \tilde{\varrho}_{\ell}\right]$ to prove the following relation:

$$
\mathfrak{D}^{j} w(s)=\tilde{\varrho}_{\ell}^{-j}\left(\mathfrak{D}^{j} \sigma(\tilde{\theta})\right)
$$

By using (1.3) on the interval $[0,1]$, we can write (1.1) as

$$
\left\{\begin{array}{l}
\mathfrak{D}^{j} \sigma(\tilde{\theta})=\tilde{\varrho}_{\ell}^{j} \mathcal{H}_{\ell}\left(s, \sigma_{\ell}(\tilde{\theta}), \tilde{\varrho}_{\ell}^{-k} \mathfrak{D}^{k} \sigma_{k}(\tilde{\theta})\right) \quad(\tilde{\theta} \in[0,1]), \\
\sigma_{\ell}(0)=0, \quad \sigma_{\ell}(1)=\sigma_{m}(1) \quad(\ell \neq m), \sum_{\ell=1}^{u} \tilde{\varrho}_{\ell}^{-1} \sigma_{\ell}^{\prime}(1)=0,
\end{array}\right.
$$

where $\sigma_{\ell}(\tilde{\theta})=w_{\ell}\left(\tilde{\varrho}_{\ell} \tilde{\theta}\right)$ and $\mathcal{H}_{k}(\tilde{\theta}, z, w)=\mathcal{H}_{\ell}\left(\tilde{\varrho}_{\ell} \tilde{\theta}, z, w\right)$ for $\ell=1,2, \ldots, u$.

Recently, Mophou et al. [25] investigated the solution of the following fractional SturmLiouville boundary value problems on a star graph:

$$
\left\{\begin{array}{l}
\mathfrak{D}_{b_{\ell}^{-1}}^{j}\left(\beta^{\ell} \mathbb{D}_{a^{+}}^{j} w^{\ell}\right)(s)+q^{\ell}(s) w^{\ell}(s)=\mathcal{H}^{\ell}(s), \quad s \in\left(a, b^{\ell}\right), \ell=1,2, \ldots, n, \\
I_{a^{+}}^{1-j} w^{\ell}\left(a^{+}\right)=I_{a^{+}}^{1-j} w^{m}\left(a^{+}\right), \quad j \neq m=1,2, \ldots, n, \\
\sum_{\ell=1}^{n} \beta^{\ell}(a) \mathbb{D}_{a^{+}}^{j} w^{\ell}\left(a^{+}\right)=0, \\
I_{a^{+}}^{1-j} w^{1}\left(b_{1}^{-}\right)=0, \\
I_{a^{+}}^{1-j} w^{\ell}\left(b_{\ell}^{-}\right)=v_{\ell}, \quad \ell=2,3, \ldots, p, \\
\beta^{\ell}\left(b_{\ell}\right) \mathbb{D}_{a^{+}}^{j} w^{\ell}\left(b_{\ell}^{-}\right)=v_{\ell}, \quad \ell=p, p+1, \ldots, n,
\end{array}\right.
$$

where $\mathbb{D}_{a^{+}}^{j}$ and $\mathfrak{D}_{b_{\ell}^{-1}}^{j}, \ell=1,2, \ldots, n$ stand, respectively, for the left Riemann-Liouville and the right Caputo fractional derivative of order $j \in(0,1) ; I_{a^{+}}^{j}$ is the Riemann-Liouville fractional integral of order $j$. The real functions $\beta^{\ell}$ and $q^{\ell}$ are defined on $\left[a, b_{\ell}\right](\ell=1,2, \ldots, n)$. The function $\mathcal{H}^{\ell}$ belongs to $L^{2}\left(a, b_{\ell}\right), \ell=1,2, \ldots, n$, and the controls $v_{\ell}, \ell=1,2, \ldots, n$ are real variables.

For the recent research in this area, we refer to [26-29] and the references therein.

In this work, we utilized the concept of the cyclohexane graph (see Fig. 2) to extend the idea of the above fractional boundary value problems to a new problem that is more generic than star graphs.

The method used in [23] and [24] for identifying the origin at boundary nodes other than the junction node $\tilde{p}_{0}$ would be insufficient since a cyclohexane graph has several junction points. As a result, we use another procedure in which we label the vertices of the preceding graph with 0 or 1 having edge length $\tilde{\varrho}_{\ell}=1$ (see Fig. 3).

Here, we examine the existence of solutions to the following problem:

$$
\left\{\begin{array}{l}
\mathfrak{D}^{j} w_{\ell}(s)=\mathcal{H}_{\ell}\left(s, w_{\ell}(s), \mathfrak{D}^{k} w_{\ell}(s), w_{\ell}^{\prime}(s)\right) \quad(s \in[0,1]) \\
\eta_{1} w_{\ell}(0)+\eta_{2} w_{\ell}(1)=\eta_{3} \int_{0}^{1} \mathfrak{D}^{j-1} w_{\ell}(\omega) d \omega \\
\eta_{1} \mathcal{D}^{k} w_{\ell}(1)+\eta_{2} \mathcal{D}^{2 k} w_{\ell}(1)=\eta_{3} \int_{0}^{1} \mathfrak{D}^{j-1} w_{\ell}(\omega) d \omega
\end{array}\right.
$$

where $\eta_{\kappa}(\kappa=1,2,3)$ are real constants with $\eta_{\kappa} \neq 0, \mathfrak{D}^{j}, \mathfrak{D}^{j-1}$, and $\mathfrak{D}^{k}$ are the Caputo fractional derivative of orders $j \in(1,2], j-1 \in(0,1]$, and $k \in(0,1)$, respectively. Also, 


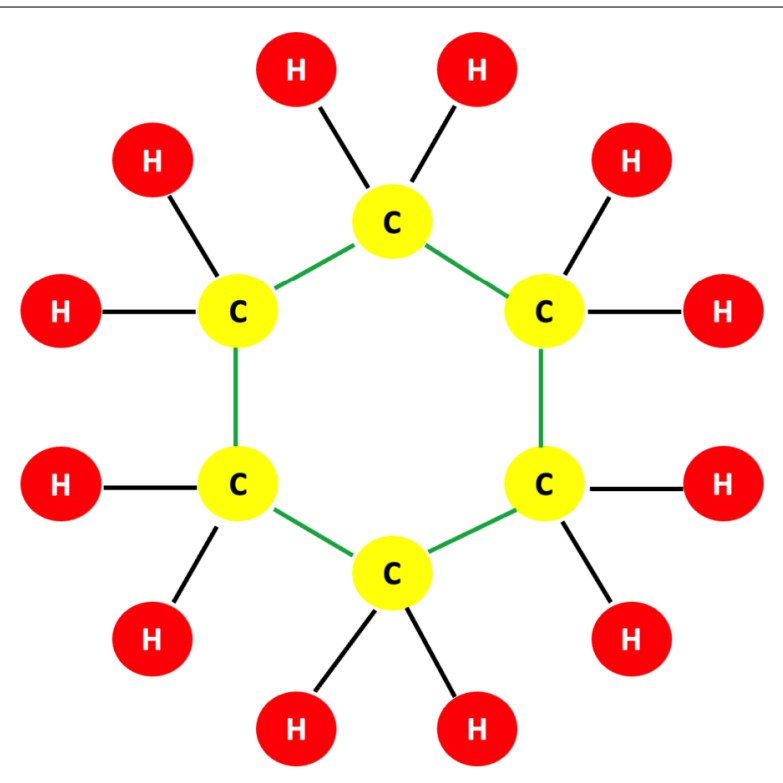

Figure 2 Chemical bond of cyclohexane compound

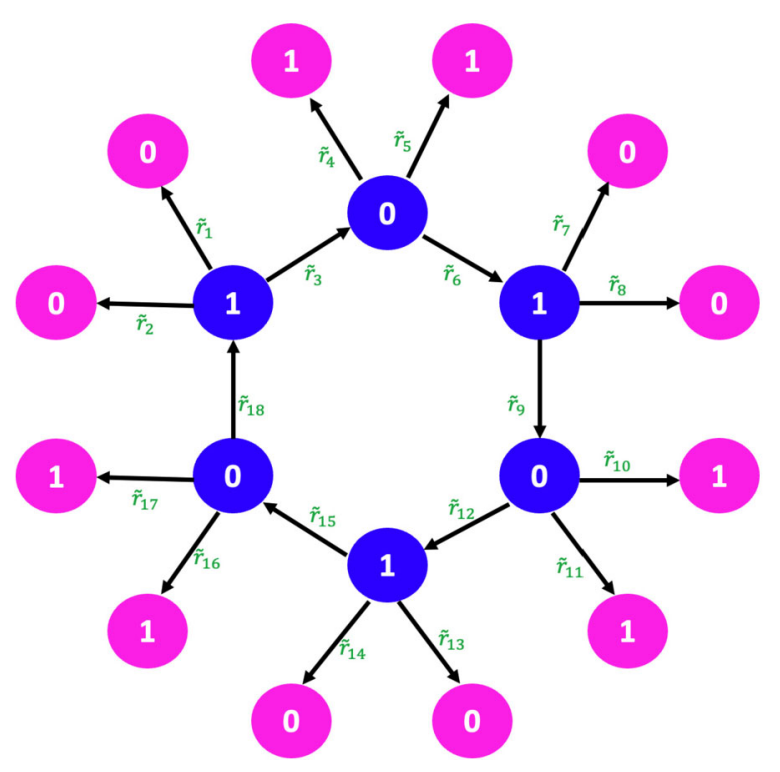

Figure 3 Cyclohexane graph with vertices 0 or 1

$\mathcal{H}_{\ell}:[0,1] \times \mathbb{R} \times \mathbb{R} \times \mathbb{R} \rightarrow \mathbb{R}$ is a given continuously differentiable function for $\ell=18$, where $\ell$ is the number of edges of the graph representation of cyclohexane compound with $\left|\tilde{r}_{k}\right|=1$. Also, $\mathcal{D}^{2 m}$ is the sequential fractional derivative discussed in [2]

$$
\left\{\begin{array}{l}
\mathcal{D}^{k} w=\mathfrak{D}^{k} w \\
\mathcal{D}^{\tau k} w=\mathcal{D}^{k} \mathcal{D}^{(\tau-1) k} w \quad(\tau=2,3, \ldots) .
\end{array}\right.
$$


Our goal is to use the relevant fixed point theorems to prove the existence of solutions to the proposed problem (1.6). Finally, an example is given to highlight the significance of our results in the particular literature.

\section{Preliminaries}

In the next sections, the subsequent outcomes will be required.

Definition 2.1 ([2]) The Caputo fractional derivative of order $j>0$ for a function $\mathcal{H} \in$ $C^{\chi}[0,+\infty)$ is defined as

$$
\mathfrak{D}^{j} \mathcal{H}(s)=\frac{1}{\Gamma(\chi-j)} \int_{0}^{s}(s-\omega)^{\chi-j-1} \mathcal{H}^{(\chi)}(\omega) d \omega \quad(\chi-1<j<\chi, \chi=[j]+1),
$$

where $[j]$ represents the integer part of $j$.

Lemma 2.2 For $p>0$, the general solution of the fractional differential equation $\mathfrak{D}^{j} w(s)=0$ is given by

$$
w(s)=z_{0}+z_{1} s+z_{2} s^{2}+\cdots+z_{n-1} s^{n-1},
$$

where $z_{k} \in \mathbb{R}, k=0,1, \ldots, n-1(n-1<j<n, n=[j]+1)$.

Also,

$$
\mathbb{I}^{p} \mathfrak{D}^{j} w(s)=w(s)+z_{0}+z_{1} s+z_{2} s^{2}+\cdots+z_{n-1} s^{n-1},
$$

for some $z_{k} \in \mathbb{R}, k=0,1, \ldots, n-1(n-1<j<n, n=[j]+1)$.

Lemma 2.3 Suppose that real-valued functions $\Upsilon_{1}, \Upsilon_{2}, \ldots, \Upsilon_{18}$ are continuous on the closed interval $[0,1]$. Then $w_{\ell}^{\star}$ is a solution for boundary value problem satisfying the boundary conditions

$$
\left\{\begin{array}{l}
\mathfrak{D}^{j} w_{\ell}(s)=\Upsilon_{\ell}(t) \quad(s \in[0,1], \ell=1,2, \ldots, 18), \\
\eta_{1} w_{\ell}(0)+\eta_{2} w_{\ell}(1)=\eta_{3} \int_{0}^{1} \mathfrak{D}^{j-1} w_{\ell}(\omega) d \omega \\
\eta_{1} \mathcal{D}^{k} w_{\ell}(1)+\eta_{2} \mathcal{D}^{2 k} w_{\ell}(1)=\eta_{3} \int_{0}^{1} \mathfrak{D}^{j-1} w_{\ell}(\omega) d \omega
\end{array}\right.
$$

if and only if $w_{\ell}^{\star}$ is a solution for the following fractional integral equation:

$$
\begin{aligned}
w_{\ell}(s)= & \int_{0}^{s} \frac{(s-\omega)^{j-1}}{\Gamma(j)} \Upsilon_{\ell}(\omega) d \omega \\
& +\eta_{3}\left(\frac{1}{\eta_{1}+\eta_{2}}+\frac{A_{0}+s}{A_{1}}\right) \int_{0}^{1} \int_{0}^{\omega} \Upsilon_{\ell}(\zeta) d \zeta d \omega \\
& -\frac{\eta_{2}}{\eta_{1}+\eta_{2}} \int_{0}^{1} \frac{(1-\omega)^{j-1}}{\Gamma(j)} \Upsilon_{\ell}(\omega) d \omega \\
& -\frac{A_{0}+s}{A_{1}}\left[\frac{\eta_{2}}{\Gamma(j-2 k)} \int_{0}^{1}(1-\omega)^{j-2 k-1} \Upsilon_{\ell}(\omega) d \omega\right. \\
& \left.+\frac{\eta_{1}}{\Gamma(j-k)} \int_{0}^{1}(1-\omega)^{j-k-1} \Upsilon_{\ell}(\omega) d \omega\right],
\end{aligned}
$$


where

$$
A_{0}=\frac{\eta_{3}-\eta_{2} \Gamma(4-j)}{\left(\eta_{1}+\eta_{2}\right) \Gamma(4-j)}, \quad A_{1}=\frac{\eta_{1}}{\Gamma(2-k)}+\frac{\eta_{2}}{\Gamma(2-2 k)}-\frac{\eta_{3}}{\Gamma(4-j)} .
$$

Proof Suppose that $w_{\ell}^{\star}$ is a solution of (2.1), where $\ell=1,2, \ldots, 18$. Then there exist constants $z_{0}^{(\ell)}, z_{1}^{(\ell)} \in \mathbb{R}$ such that

$$
w_{\ell}^{\star}(s)=\int_{0}^{s} \frac{(s-\omega)^{j-1}}{\Gamma(j)} \Upsilon_{\ell}(\omega) d \omega+z_{0}^{(\ell)}+z_{1}^{(\ell)} s
$$

Using the boundary conditions for (2.1), we have

$$
\begin{aligned}
z_{0}^{(\ell)}= & \eta_{3}\left(\frac{1}{\eta_{1}+\eta_{2}}+\frac{A_{0}}{A_{1}}\right) \int_{0}^{1} \int_{0}^{\omega} \Upsilon_{\ell}(\zeta) d \zeta d \omega \\
& -\frac{\eta_{2}}{\eta_{1}+\eta_{2}} \int_{0}^{1} \frac{(1-\omega)^{j-1}}{\Gamma(j)} \Upsilon_{\ell}(\omega) d \omega \\
& -\frac{A_{0}}{A_{1}}\left[\frac{\eta_{2}}{\Gamma(j-2 k)} \int_{0}^{1}(1-\omega)^{j-2 k-1} \Upsilon_{\ell}(\omega) d \omega\right. \\
& \left.+\frac{\eta_{1}}{\Gamma(j-k)} \int_{0}^{1}(1-\omega)^{j-k-1} \Upsilon_{\ell}(\omega) d \omega\right], \\
z_{1}^{(\ell)}= & \frac{\eta_{3}}{A_{1}} \int_{0}^{1} \int_{0}^{\omega} \Upsilon_{\ell}(\zeta) d \zeta d \omega-\frac{\eta_{2}}{A_{1}} \int_{0}^{1} \frac{(1-\omega)^{j-2 k-1}}{\Gamma(j-2 k)} \Upsilon_{\ell}(\omega) d \omega \\
& -\frac{\eta_{1}}{A_{1}} \int_{0}^{1} \frac{(1-\omega)^{j-k-1}}{\Gamma(j-k)} \Upsilon_{\ell}(\omega) d \omega .
\end{aligned}
$$

Substituting the values of $z_{0}^{(\ell)}$ and $z_{1}^{(\ell)}$ into (2.3), we obtain the solution (2.2). With regard to the converse statement, it is self-evident that $w_{\ell}^{\star}$ is a solution for $(2.1)$ when it is a solution for an integral equation (2.3).

The fixed point theorems of Schaefer and Krasnoselskii are now presented.

Theorem 2.4 ([30]) If $\mathcal{T}$ is a completely continuous, that is, $\mathcal{T}$ is continuous and totally bounded, self-operator on a Banach space $\mathcal{C}$, then either $\{a \in \mathcal{C}: a=b \mathcal{T}$ a for some $b \in$ $(0,1)\}$ is unbounded or $\mathcal{T}$ has a fixed point.

Theorem 2.5 ([30]) Let $\mathcal{O}$ be a closed convex and nonempty subset of a Banach space $\mathcal{C}$ and $\mathcal{T}_{1}, \mathcal{T}_{2}: \mathcal{O} \rightarrow \mathcal{C}$ be two operators satisfying the following conditions:

1. $\mathcal{T}_{1} k+\mathcal{T}_{2} k^{\prime} \in \mathcal{O}$ for all $k, k^{\prime} \in \mathcal{O}$;

2. $\mathcal{T}_{1}$ is compact and continuous on $\mathcal{O}$;

3. $\mathcal{T}_{2}$ is a Banach contraction mapping on $\mathcal{O}$, that is, there is $\delta \in[0,1)$ such that

$$
\left\|\mathcal{T}_{2} k-\mathcal{T}_{2} k^{\prime}\right\| \leq \delta\left\|k-k^{\prime}\right\|
$$

for all $k, k^{\prime} \in \mathcal{O}$. Then $\mathcal{T}_{1}+\mathcal{T}_{2}$ has a fixed point 


\section{Main results}

We define the Banach space $\mathcal{C}_{\ell}=\left\{w_{\ell}: w_{\ell}, \mathfrak{D}^{k} w_{\ell}, w_{\ell}^{\prime} \in C[0,1]\right\}$ with the norm

$$
\left\|w_{\ell}\right\|_{\mathcal{C}_{\ell}}=\sup _{s \in[0,1]}\left|w_{\ell}(s)\right|+\sup _{s \in[0,1]}\left|\mathfrak{D}^{k} w_{\ell}(s)\right|+\sup _{s \in[0,1]}\left|w_{\ell}^{\prime}(s)\right|
$$

for $\ell=1,2, \ldots, 18$. It is obvious that the product space $\mathcal{C}=\mathcal{C}_{1} \times \mathcal{C}_{2} \times \cdots \times \mathcal{C}_{18}$ is a Banach space, where the norm is defined by

$$
\left\|w=\left(w_{1}, w_{2}, \ldots, w_{18}\right)\right\|_{\mathcal{C}}=\sum_{\ell=1}^{18}\left\|w_{\ell}\right\|_{\mathcal{C}_{\ell}} .
$$

In order to apply Lemma 2.3, we introduce the operator $\mathcal{T}: \mathcal{C} \rightarrow \mathcal{C}$ by

$$
\mathcal{T}\left(w_{1}, w_{2}, \ldots, w_{18}\right)(s):=\left(\mathcal{T}_{1}\left(w_{1}, w_{2}, \ldots, w_{18}\right), \ldots, \mathcal{T}_{18}\left(w_{1}, w_{2}, \ldots, w_{18}\right)(s)\right)
$$

where

$$
\begin{aligned}
\mathcal{T}_{\ell}\left(w_{1}, w_{2}, \ldots, w_{18}\right)(s) \\
=\int_{0}^{s} \frac{(s-\omega)^{j-1}}{\Gamma(j)} \mathcal{H}_{\ell}\left(\omega, w_{\ell}(\omega), \mathfrak{D}^{k} w_{\ell}(\omega), w_{\ell}^{\prime}(\omega)\right) d \omega \\
\quad+\eta_{3}\left(\frac{1}{\eta_{1}+\eta_{2}}+\frac{A_{0}+s}{A_{1}}\right) \int_{0}^{1} \int_{0}^{\omega} \mathcal{H}_{\ell}\left(\zeta, w_{\ell}(\zeta), \mathfrak{D}^{k} w_{\ell}(\zeta), w_{\ell}^{\prime}(\zeta)\right) d \zeta d \omega \\
\quad-\frac{\eta_{2}}{\eta_{1}+\eta_{2}} \int_{0}^{1} \frac{(1-\omega)^{j-1}}{\Gamma(j)} \mathcal{H}_{\ell}\left(\omega, w_{\ell}(\omega), \mathfrak{D}^{k} w_{\ell}(\omega), w_{\ell}^{\prime}(\omega)\right) d \omega \\
\quad-\frac{A_{0}+s}{A_{1}}\left[\frac{\eta_{2}}{\Gamma(j-2 k)} \int_{0}^{1}(1-\omega)^{j-2 k-1} \mathcal{H}_{\ell}\left(\omega, w_{\ell}(\omega), \mathfrak{D}^{k} w_{\ell}(\omega), w_{\ell}^{\prime}(\omega)\right) d \omega\right. \\
\left.\quad+\frac{\eta_{1}}{\Gamma(j-k)} \int_{0}^{1}(1-\omega)^{j-k-1} \mathcal{H}_{\ell}\left(\omega, w_{\ell}(\omega), \mathfrak{D}^{k} w_{\ell}(\omega), w_{\ell}^{\prime}(\omega)\right) d \omega\right]
\end{aligned}
$$

for all $s \in[0,1]$ and $w_{\ell} \in \mathcal{C}_{\ell}$.

To simplify calculations, we shall use the following notation:

$$
\begin{aligned}
A_{0}= & \frac{\eta_{3}-\eta_{2} \Gamma(4-j)}{\left(\eta_{1}+\eta_{2}\right) \Gamma(4-j)}, \\
A_{1}= & {\left[\frac{\eta_{1}}{\Gamma(2-k)}+\frac{\eta_{2}}{\Gamma(2-2 k)}-\frac{\eta_{3}}{\Gamma(4-j)}\right] \neq 0, } \\
\mathcal{F}_{0}^{*}= & \frac{1}{\Gamma(j+1)}+\left(\frac{1+\left|A_{0}\right|}{\left|A_{1}\right|}\right) \frac{\left|\eta_{1}\right|}{\Gamma(j-k+1)}+\left(\frac{1+\left|A_{0}\right|}{\left|A_{1}\right|}+\frac{1}{\left|\eta_{1}+\eta_{2}\right|}\right) \frac{\left|\eta_{3}\right|}{2} \\
& +\left(\frac{1+\left|A_{0}\right|}{\left|A_{1}\right| \Gamma(j-2 k+1)}+\frac{1}{\left|\eta_{1}+\eta_{2}\right| \Gamma(j+1)}\right)\left|\eta_{2}\right|, \\
\mathcal{F}_{1}^{*}= & \frac{1}{\Gamma(j-k+1)}+\frac{1}{\Gamma(2-k)\left|A_{1}\right|} \\
& \times\left(\frac{\left|\eta_{1}\right|}{\Gamma(j-k+1)}+\frac{\left|\eta_{2}\right|}{\Gamma(j-2 k+1)}+\frac{\left|\eta_{3}\right|}{2}\right),
\end{aligned}
$$




$$
\begin{aligned}
\mathcal{F}_{2}^{*}= & \frac{1}{\Gamma(j)}+\frac{1}{\left|A_{1}\right|}\left(\frac{\left|\eta_{1}\right|}{\Gamma(j-k+1)}+\frac{\left|\eta_{2}\right|}{\Gamma(j-2 k+1)}+\frac{\left|\eta_{3}\right|}{2}\right), \\
\mathcal{V}_{0}^{*}= & \left(\frac{1+\left|A_{0}\right|}{\left|A_{1}\right|}\right) \frac{\left|\eta_{1}\right|}{\Gamma(j-k+1)}+\left(\frac{1+\left|A_{0}\right|}{\left|A_{1}\right|}+\frac{1}{\left|\eta_{1}+\eta_{2}\right|}\right) \frac{\left|\eta_{3}\right|}{2} \\
& +\left(\frac{1+\left|A_{0}\right|}{\left|A_{1}\right| \Gamma(j-2 k+1)}+\frac{1}{\left|\eta_{1}+\eta_{2}\right| \Gamma(j+1)}\right)\left|\eta_{2}\right|, \\
\mathcal{V}_{1}^{*}= & \frac{1}{\Gamma(2-k)\left|A_{1}\right|}\left(\frac{\left|\eta_{1}\right|}{\Gamma(j-k+1)}+\frac{\left|\eta_{2}\right|}{\Gamma(j-2 k+1)}+\frac{\left|\eta_{3}\right|}{2}\right), \\
\mathcal{V}_{2}^{*}= & \frac{1}{\left|A_{1}\right|}\left(\frac{\left|\eta_{1}\right|}{\Gamma(j-k+1)}+\frac{\left|\eta_{2}\right|}{\Gamma(j-2 k+1)}+\frac{\left|\eta_{3}\right|}{2}\right) .
\end{aligned}
$$

Theorem 3.1 Consider the fractional boundary value problem (1.6). Suppose that $\mathcal{H}_{1}, \mathcal{H}_{2}$, $\ldots, \mathcal{H}_{18}:[0,1] \times \mathbb{R} \times \mathbb{R} \times \mathbb{R} \rightarrow \mathbb{R}$ are continuous functions and there exist constants $\Xi_{\ell}>$ 0 for all $\ell=1,2, \ldots, 18$ such that $\left|\mathcal{H}_{\ell}(s, w, \tilde{w}, \tilde{\tilde{w}})\right| \leq \Xi_{\ell}$ for all $w, \tilde{w}, \tilde{\tilde{w}} \in \mathbb{R}, s \in[0,1]$. Then problem (1.6) has a solution.

Proof It is obvious from the implication of (3.2) that the fixed points of $\mathcal{T}$ defined by (3.1) exist if and only if (1.6) has a solution. To prove this, we first show that $\mathcal{T}$ is completely continuous.

As $\mathcal{H}_{1}, \mathcal{H}_{2}, \ldots, \mathcal{H}_{18}$ are continuous, $\mathcal{T}: \mathcal{C} \rightarrow \mathcal{C}$ is continuous, too. Let $\mathcal{O} \in \mathcal{C}$ be a bounded set and $w=\left(w_{1}, w_{2}, \ldots, w_{18}\right) \in \mathcal{C}$, so that for each $s \in[0,1]$ we have

$$
\begin{aligned}
& \left|\left(\mathcal{T}_{\ell} w\right)(s)\right| \\
& \leq \int_{0}^{s} \frac{(s-\omega)^{j-1}}{\Gamma(j)}\left|\mathcal{H}_{\ell}\left(\omega, w_{\ell}(\omega), \mathfrak{D}^{k} w_{\ell}(\omega), w_{\ell}^{\prime}(\omega)\right)\right| d \omega \\
& +\left|\eta_{3}\right|\left(\frac{1}{\left|\eta_{1}+\eta_{2}\right|}+\frac{\left|A_{0}\right|+s}{\left|A_{1}\right|}\right) \int_{0}^{1} \int_{0}^{\omega}\left|\mathcal{H}_{\ell}\left(\zeta, w_{\ell}(\zeta), \mathfrak{D}^{k} w_{\ell}(\zeta), w_{\ell}^{\prime}(\zeta)\right)\right| d \zeta d \omega \\
& +\frac{\left|\eta_{2}\right|}{\left|\eta_{1}+\eta_{2}\right|} \int_{0}^{1} \frac{(1-\omega)^{j-1}}{\Gamma(j)}\left|\mathcal{H}_{\ell}\left(\omega, w_{\ell}(\omega), \mathfrak{D}^{k} w_{\ell}(\omega), w_{\ell}^{\prime}(\omega)\right)\right| d \omega \\
& +\frac{\left|A_{0}\right|+s}{\left|A_{1}\right|}\left[\frac{\left|\eta_{2}\right|}{\Gamma(j-2 k)} \int_{0}^{1}(1-\omega)^{j-2 k-1}\left|\mathcal{H}_{\ell}\left(\omega, w_{\ell}(\omega), \mathfrak{D}^{k} w_{\ell}(\omega), w_{\ell}^{\prime}(\omega)\right)\right| d \omega\right. \\
& \left.+\frac{\left|\eta_{1}\right|}{\Gamma(j-k)} \int_{0}^{1}(1-\omega)^{j-k-1}\left|\mathcal{H}_{\ell}\left(\omega, w_{\ell}(\omega), \mathfrak{D}^{k} w_{\ell}(\omega), w_{\ell}^{\prime}(\omega)\right)\right| d \omega\right] \\
& \leq \Xi_{\ell}\left[\frac{1}{\Gamma(j+1)}+\left(\frac{1+\left|A_{0}\right|}{\left|A_{1}\right|}\right) \frac{\left|\eta_{1}\right|}{\Gamma(j-k+1)}+\left(\frac{1+\left|A_{0}\right|}{\left|A_{1}\right|}+\frac{1}{\left|\eta_{1}+\eta_{2}\right|}\right) \frac{\left|\eta_{3}\right|}{2}\right. \\
& \left.+\left(\frac{1+\left|A_{0}\right|}{\left|A_{1}\right| \Gamma(j-2 k+1)}+\frac{1}{\left|\eta_{1}+\eta_{2}\right| \Gamma(j+1)}\right)\left|\eta_{2}\right|\right] \\
& =\Xi_{\ell} \mathcal{F}_{0}^{*} \text {, }
\end{aligned}
$$

where $\mathcal{F}_{0}^{*}$ is given in (3.5). Also,

$$
\begin{aligned}
& \left|\left(\mathfrak{D}^{k} \mathcal{T}_{\ell} w\right)(s)\right| \\
& \quad \leq \int_{0}^{s} \frac{(s-\omega)^{j-k-1}}{\Gamma(j-k)}\left|\mathcal{H}_{\ell}\left(\omega, w_{\ell}(\omega), \mathfrak{D}^{k} w_{\ell}(\omega), w_{\ell}^{\prime}(\omega)\right)\right| d \omega
\end{aligned}
$$




$$
\begin{aligned}
& +\frac{\left|\eta_{3}\right| s^{1-k}}{\Gamma(2-k)\left|A_{1}\right|} \int_{0}^{1} \int_{0}^{\omega}\left|\mathcal{H}_{\ell}\left(\zeta, w_{\ell}(\zeta), \mathfrak{D}^{k} w_{\ell}(\zeta), w_{\ell}^{\prime}(\zeta)\right)\right| d \zeta d \omega \\
& +\frac{s^{1-k}}{\Gamma(2-k)\left|A_{1}\right|}\left[\frac{\left|\eta_{2}\right|}{\Gamma(j-2 k)} \int_{0}^{1}(1-\omega)^{j-2 k-1}\left|\mathcal{H}_{\ell}\left(\omega, w_{\ell}(\omega), \mathfrak{D}^{k} w_{\ell}(\omega), w_{\ell}^{\prime}(\omega)\right)\right| d \omega\right. \\
& \left.+\frac{\left|\eta_{1}\right|}{\Gamma(j-k)} \int_{0}^{1}(1-\omega)^{j-k-1}\left|\mathcal{H}_{\ell}\left(\omega, w_{\ell}(\omega), \mathfrak{D}^{k} w_{\ell}(\omega), w_{\ell}^{\prime}(\omega)\right)\right| d \omega\right] \\
& \leq \Xi_{\ell}\left[\frac{1}{\Gamma(j-k+1)}+\frac{1}{\Gamma(2-k)\left|A_{1}\right|}\left(\frac{\left|\eta_{1}\right|}{\Gamma(j-k+1)}+\frac{\left|\eta_{2}\right|}{\Gamma(j-2 k+1)}+\frac{\left|\eta_{3}\right|}{2}\right)\right] \\
& =\Xi_{\ell} \mathcal{F}_{1}^{*},
\end{aligned}
$$

and

$$
\begin{aligned}
\left|\left(\mathcal{T}_{\ell}^{\prime} w\right)(s)\right| \leq & \int_{0}^{s} \frac{(s-\omega)^{j-2}}{\Gamma(j-1)}\left|\mathcal{H}_{\ell}\left(\omega, w_{\ell}(\omega), \mathfrak{D}^{k} w_{\ell}(\omega), w_{\ell}^{\prime}(\omega)\right)\right| d \omega \\
& +\frac{\left|\eta_{3}\right|}{\left|A_{1}\right|} \int_{0}^{1} \int_{0}^{\omega}\left|\mathcal{H}_{\ell}\left(\zeta, w_{\ell}(\zeta), \mathfrak{D}^{k} w_{\ell}(\zeta), w_{\ell}^{\prime}(\zeta)\right)\right| d \zeta d \omega \\
& +\frac{1}{\left|A_{1}\right|}\left[\frac{\left|\eta_{2}\right|}{\Gamma(j-2 k)} \int_{0}^{1}(1-\omega)^{j-2 k-1}\left|\mathcal{H}_{\ell}\left(\omega, w_{\ell}(\omega), \mathfrak{D}^{k} w_{\ell}(\omega), w_{\ell}^{\prime}(\omega)\right)\right| d \omega\right. \\
& \left.+\frac{\left|\eta_{1}\right|}{\Gamma(j-k)} \int_{0}^{1}(1-\omega)^{j-k-1}\left|\mathcal{H}_{\ell}\left(\omega, w_{\ell}(\omega), \mathfrak{D}^{k} w_{\ell}(\omega), w_{\ell}^{\prime}(\omega)\right)\right| d \omega\right] \\
\leq & \Xi_{\ell}\left[\frac{1}{\Gamma(j)}+\frac{1}{\left|A_{1}\right|}\left(\frac{\left|\eta_{1}\right|}{\Gamma(j-k+1)}+\frac{\left|\eta_{2}\right|}{\Gamma(j-2 k+1)}+\frac{\left|\eta_{3}\right|}{2}\right)\right] \\
& =\Xi_{\ell} \mathcal{F}_{2}^{*}
\end{aligned}
$$

for all $s \in[0,1]$, where $\mathcal{F}_{1}^{*}, \mathcal{F}_{2}^{*}$ are defined in (3.6) and (3.7), respectively. Therefore

$$
\left\|\left(\mathcal{T}_{\ell} w\right)(s)\right\|_{\mathcal{C}_{\ell}} \leq \Xi_{\ell}\left(\mathcal{F}_{0}^{*}+\mathcal{F}_{1}^{*}+\mathcal{F}_{2}^{*}\right)
$$

Hence,

$$
\|(\mathcal{T} w)(s)\|_{\mathcal{C}}=\sum_{\ell=1}^{18}\left\|\left(\mathcal{T}_{\ell} w\right)(s)\right\|_{\mathcal{C}_{\ell}} \leq \sum_{\ell=1}^{18} \Xi_{\ell}\left(\mathcal{F}_{0}^{*}+\mathcal{F}_{1}^{*}+\mathcal{F}_{2}^{*}\right)<\infty
$$

which shows that $\mathcal{T}$ is uniformly bounded.

Now, we have to prove that $\mathcal{T}$ is equicontinuous. As for this purpose, let $w=\left(w_{1}, w_{2}, \ldots\right.$, $\left.w_{18}\right) \in \mathcal{O}$ and $s_{1}, s_{2} \in[0,1]$ with $s_{1}<s_{2}$. Then, we have

$$
\begin{aligned}
& \left|\left(\mathcal{T}_{\ell} w\right)\left(s_{2}\right)-\left(\mathcal{T}_{\ell} w\right)\left(s_{1}\right)\right| \\
& =\int_{0}^{s_{1}} \frac{\left(s_{2}-\omega\right)^{j-1}-\left(s_{1}-\omega\right)^{j-1}}{\Gamma(j)}\left|\mathcal{H}_{\ell}\left(\omega, w_{\ell}(\omega), \mathfrak{D}^{k} w_{\ell}(\omega), w_{\ell}^{\prime}(\omega)\right)\right| d \omega \\
& \quad+\int_{s_{1}}^{s_{2}} \frac{\left(s_{2}-\omega\right)^{j-1}}{\Gamma(j)}\left|\mathcal{H}_{\ell}\left(\omega, w_{\ell}(\omega), \mathfrak{D}^{k} w_{\ell}(\omega), w_{\ell}^{\prime}(\omega)\right)\right| d \omega \\
& \quad+\frac{\left|\eta_{3}\right|\left(s_{2}-s_{1}\right)}{\left|A_{1}\right|} \int_{0}^{1} \int_{0}^{\omega}\left|\mathcal{H}_{\ell}\left(\zeta, w_{\ell}(\zeta), \mathfrak{D}^{k} w_{\ell}(\zeta), w_{\ell}^{\prime}(\zeta)\right)\right| d \zeta d \omega
\end{aligned}
$$




$$
\begin{aligned}
& +\frac{\left(s_{2}-s_{1}\right)}{\left|A_{1}\right|}\left[\frac{\left|\eta_{2}\right|}{\Gamma(j-2 k)} \int_{0}^{1}(1-\omega)^{j-2 k-1}\left|\mathcal{H}_{\ell}\left(\omega, w_{\ell}(\omega), \mathfrak{D}^{k} w_{\ell}(\omega), w_{\ell}^{\prime}(\omega)\right)\right| d \omega\right. \\
& \left.+\frac{\left|\eta_{1}\right|}{\Gamma(j-k)} \int_{0}^{1}(1-\omega)^{j-k-1}\left|\mathcal{H}_{\ell}\left(\omega, w_{\ell}(\omega), \mathfrak{D}^{k} w_{\ell}(\omega), w_{\ell}^{\prime}(\omega)\right)\right| d \omega\right] .
\end{aligned}
$$

It is clear that if $s_{1} \rightarrow s_{2}$ then, independently, the right-hand side of the above expression converges to zero. Also,

$$
\lim _{s_{1} \rightarrow s_{2}}\left|\left(\mathfrak{D}^{k} \mathcal{T}_{\ell} w\right)\left(s_{2}\right)-\left(\mathfrak{D}^{k} \mathcal{T}_{\ell} w\right)\left(s_{1}\right)\right|=0, \quad \lim _{s_{1} \rightarrow s_{2}}\left|\left(\mathcal{T}_{\ell}^{\prime} w\right)\left(s_{2}\right)-\left(\mathcal{T}_{\ell}^{\prime} w\right)\left(s_{1}\right)\right|=0
$$

As a result $\left\|(\mathcal{T} w)\left(s_{2}\right)-(\mathcal{T} w)\left(s_{1}\right)\right\|_{\mathcal{C}} \rightarrow 0$ as $s_{1} \rightarrow s_{2}$. This proves that $\mathcal{T}$ is equicontinuous on $\mathcal{C}=\mathcal{C}_{1} \times \mathcal{C}_{2} \times \cdots \times \mathcal{C}_{18}$. Now, the Arzela-Ascoli theorem implies the complete continuity of the operator.

Now, we define a subset $\Theta$ of $\mathcal{C}$ by

$$
\Theta:=\left\{\left(w_{1}, w_{2}, \ldots, w_{18}\right) \in \mathcal{C}:\left(w_{1}, w_{2}, \ldots, w_{18}\right)=b \mathcal{T}\left(w_{1}, w_{2}, \ldots, w_{18}\right), b \in(0,1)\right\} .
$$

Here, we shall show that $\Theta$ is bounded. For this, let $\left(w_{1}, w_{2}, \ldots, w_{18}\right) \in \Theta$. Then, we can write $\left(w_{1}, w_{2}, \ldots, w_{18}\right)=b \mathcal{T}\left(w_{1}, w_{2}, \ldots, w_{18}\right)$, and so $w_{\ell}(s)=b \mathcal{T}_{\ell}\left(w_{1}, w_{2}, \ldots, w_{18}\right)$, for all $s \in$ $[0,1]$ and $\ell=1,2, \ldots, 18$. Thus,

$$
\begin{aligned}
\left|w_{\ell}(s)\right| \leq & b\left[\int_{0}^{s} \frac{(s-\omega)^{j-1}}{\Gamma(j)}\left|\mathcal{H}_{\ell}\left(\omega, w_{\ell}(\omega), \mathfrak{D}^{k} w_{\ell}(\omega), w_{\ell}^{\prime}(\omega)\right)\right| d \omega\right. \\
& +\left|\eta_{3}\right|\left(\frac{1}{\left|\eta_{1}+\eta_{2}\right|}+\frac{\left|A_{0}\right|+s}{\left|A_{1}\right|}\right) \int_{0}^{1} \int_{0}^{\omega}\left|\mathcal{H}_{\ell}\left(\zeta, w_{\ell}(\zeta), \mathfrak{D}^{k} w_{\ell}(\zeta), w_{\ell}^{\prime}(\zeta)\right)\right| d \zeta d \omega \\
& +\frac{\left|\eta_{2}\right|}{\left|\eta_{1}+\eta_{2}\right|} \int_{0}^{1} \frac{(1-\omega)^{j-1}}{\Gamma(j)}\left|\mathcal{H}_{\ell}\left(\omega, w_{\ell}(\omega), \mathfrak{D}^{k} w_{\ell}(\omega), w_{\ell}^{\prime}(\omega)\right)\right| d \omega \\
& +\frac{\left|A_{0}\right|+s}{\left|A_{1}\right|}\left[\frac{\left|\eta_{2}\right|}{\Gamma(j-2 k)} \int_{0}^{1}(1-\omega)^{j-2 k-1}\left|\mathcal{H}_{\ell}\left(\omega, w_{\ell}(\omega), \mathfrak{D}^{k} w_{\ell}(\omega), w_{\ell}^{\prime}(\omega)\right)\right| d \omega\right. \\
& \left.+\frac{\left|\eta_{1}\right|}{\Gamma(j-k)} \int_{0}^{1}(1-\omega)^{j-k-1}\left|\mathcal{H}_{\ell}\left(\omega, w_{\ell}(\omega), \mathfrak{D}^{k} w_{\ell}(\omega), w_{\ell}^{\prime}(\omega)\right)\right| d \omega\right] \\
\leq & b \Xi_{\ell} \mathcal{F}_{0}^{*} .
\end{aligned}
$$

By similar computations, we have $\left|\mathfrak{D}^{k} w_{\ell}(s)\right| \leq b \Xi_{\ell} \mathcal{F}_{1}^{*},\left|w_{\ell}^{\prime}(s)\right| \leq b \Xi_{\ell} \mathcal{F}_{2}^{*}$, where $\mathcal{F}_{0}^{*}, \ldots, \mathcal{F}_{2}^{*}$ are given in (3.5)-(3.7). Hence

$$
\|w\|_{\mathcal{C}}=\sum_{\ell=1}^{18}\left\|w_{\ell}\right\|_{\mathcal{C}_{\ell}} \leq b \sum_{\ell=1}^{18} \Xi_{\ell}\left(\mathcal{F}_{0}^{*}+\mathcal{F}_{1}^{*}+\mathcal{F}_{2}^{*}\right)<\infty
$$

which shows the boundedness of $\Theta$. Now, using Theorem 2.4 and Lemma 2.3, we can claim that $\mathcal{T}$ has a fixed point in $\mathcal{C}$. This demonstrates that the fractional boundary value problem (1.6) does indeed have a solution.

We shall now examine the solution of the fractional boundary value problem (1.6) by applying various conditions. 
Theorem 3.2 Consider the fractional boundary value problem (1.6). Assume that $\mathcal{H}_{1}, \mathcal{H}_{2}$, $\ldots, \mathcal{H}_{18}:[0,1] \times \mathbb{R} \times \mathbb{R} \times \mathbb{R} \rightarrow \mathbb{R}$ are continuous functions and there exist bounded continuous functions $\mathcal{S}_{1}, \mathcal{S}_{2}, \ldots, \mathcal{S}_{18}:[0,1] \rightarrow \mathbb{R}, \mathcal{W}_{1}, \mathcal{W}_{2}, \ldots, \mathcal{W}_{18}:[0,1] \rightarrow[0, \infty)$ and nondecreasing continuous functions $\mathcal{P}_{1}, \mathcal{P}_{2}, \ldots, \mathcal{P}_{18}:[0,1] \rightarrow[0, \infty)$ such that

$$
\left|\mathcal{H}_{\ell}(s, w, \tilde{w}, \tilde{\tilde{w}})\right| \leq \mathcal{W}_{\ell}(s) \mathcal{P}_{\ell}(|w|+|\tilde{w}|+|\tilde{\tilde{w}}|)
$$

and

$$
\left|\mathcal{H}_{\ell}\left(s, w_{1}, w_{2}, w_{3}\right)-\mathcal{H}_{\ell}\left(s, \tilde{w}_{1}, \tilde{w}_{2}, \tilde{w}_{3}\right)\right| \leq \mathcal{S}_{\ell}(s)\left(\left|w_{1}-\tilde{w}_{1}\right|+\left|w_{2}-\tilde{w}_{2}\right|+\left|w_{3}-\tilde{w}_{3}\right|\right)
$$

for all $s \in[0,1], w_{1}, w_{2}, w_{3}, \tilde{w}_{1}, \tilde{w}_{2}, \tilde{w}_{3} \in \mathbb{R}$ and $\ell=1,2, \ldots, 18$. If

$$
\mathcal{F}:=\left(\mathcal{V}_{0}^{*}+\mathcal{V}_{1}^{*}+\mathcal{V}_{2}^{*}\right) \sum_{\ell=1}^{18}\left\|\mathcal{S}_{\ell}\right\|<1
$$

then (1.6) has a solution, where $\left\|\mathcal{S}_{\ell}\right\|=\sup _{s \in[0,1]}\left|\mathcal{S}_{\ell}(s)\right|$ and the constants $\mathcal{V}_{0}^{*}, \ldots, \mathcal{V}_{2}^{*}$ are given in (3.8)-(3.10), respectively.

Proof First, we put $\left\|\mathcal{W}_{\ell}\right\|=\sup _{s \in[0,1]}\left|\mathcal{W}_{\ell}(s)\right|$ and choose a suitable real constant $\kappa_{\ell}$ such that

$$
\kappa_{\ell} \geq \sum_{\ell=1}^{18} \mathcal{P}_{\ell}\left(\left\|w_{\ell}\right\|_{\mathcal{C}_{\ell}}\right)\left\|\mathcal{W}_{\ell}\right\|\left\{\mathcal{F}_{0}^{*}+\mathcal{F}_{1}^{*}+\mathcal{F}_{2}^{*}\right\}
$$

where $\mathcal{F}_{0}^{*}, \ldots, \mathcal{F}_{2}^{*}$ are given in (3.5)-(3.7). We define a set

$$
\mathcal{O}_{\kappa_{\ell}}:=\left\{w=\left(w_{1}, w_{2}, \ldots, w_{18}\right) \in \mathcal{C}:\|w\|_{\mathcal{C}} \leq \kappa_{\ell}\right\}
$$

where $\kappa_{\ell}$ is defined in (3.11). It is obvious that $\mathcal{O}_{\kappa_{\ell}}$ is a nonempty closed bounded and convex subset of $\mathcal{C}=\mathcal{C}_{1} \times \mathcal{C}_{2} \times \cdots \times \mathcal{C}_{18}$. Now, we define $\mathcal{T}_{1}$ and $\mathcal{T}_{2}$ on $\mathcal{O}_{\kappa \ell}$ by

$$
\begin{aligned}
& \mathcal{T}_{1}\left(w_{1}, w_{2}, \ldots, w_{18}\right)(s):=\left(\mathcal{T}_{1}^{(1)}\left(w_{1}, w_{2}, \ldots, w_{18}\right)(s), \ldots, \mathcal{T}_{1}^{(18)}\left(w_{1}, w_{2}, \ldots, w_{18}\right)(s)\right), \\
& \mathcal{T}_{2}\left(w_{1}, w_{2}, \ldots, w_{18}\right)(s):=\left(\mathcal{T}_{2}^{(1)}\left(w_{1}, w_{2}, \ldots, w_{18}\right)(s), \ldots, \mathcal{T}_{2}^{(18)}\left(w_{1}, w_{2}, \ldots, w_{18}\right)(s)\right),
\end{aligned}
$$

where

$$
\left(\mathcal{T}_{1}^{(\ell)} w\right)(s)=\int_{0}^{s} \frac{(s-\omega)^{j-1}}{\Gamma(j)} \mathcal{H}_{\ell}\left(\omega, w_{\ell}(\omega), \mathfrak{D}^{k} w_{\ell}(\omega), w_{\ell}^{\prime}(\omega)\right) d \omega
$$

and

$$
\begin{aligned}
& \left(\mathcal{T}_{2}^{(\ell)} w\right)(s) \\
& =\eta_{3}\left(\frac{1}{\eta_{1}+\eta_{2}}+\frac{A_{0}+s}{A_{1}}\right) \int_{0}^{1} \int_{0}^{\omega} \mathcal{H}_{\ell}\left(\zeta, w_{\ell}(\zeta), \mathfrak{D}^{k} w_{\ell}(\zeta), w_{\ell}^{\prime}(\zeta)\right) d \zeta d \omega \\
& \quad-\frac{\eta_{2}}{\eta_{1}+\eta_{2}} \int_{0}^{1} \frac{(1-\omega)^{j-1}}{\Gamma(j)} \mathcal{H}_{\ell}\left(\omega, w_{\ell}(\omega), \mathfrak{D}^{k} w_{\ell}(\omega), w_{\ell}^{\prime}(\omega)\right) d \omega
\end{aligned}
$$




$$
\begin{aligned}
& -\frac{A_{0}+s}{A_{1}}\left[\frac{\eta_{2}}{\Gamma(j-2 k)} \int_{0}^{1}(1-\omega)^{j-2 k-1} \mathcal{H}_{\ell}\left(\omega, w_{\ell}(\omega), \mathfrak{D}^{k} w_{\ell}(\omega), w_{\ell}^{\prime}(\omega)\right) d \omega\right. \\
& \left.+\frac{\eta_{1}}{\Gamma(j-k)} \int_{0}^{1}(1-\omega)^{j-k-1} \mathcal{H}_{\ell}\left(\omega, w_{\ell}(\omega), \mathfrak{D}^{k} w_{\ell}(\omega), w_{\ell}^{\prime}(\omega)\right) d \omega\right]
\end{aligned}
$$

for all $s \in[0,1]$ and $w=\left(w_{1}, w_{2}, \ldots, w_{18}\right) \in \mathcal{O}_{\kappa_{\ell}}$. Let

$$
\tilde{\mathcal{P}}_{\ell}=\sup _{w_{\ell} \in \mathcal{C}_{\ell}} \mathcal{P}_{\ell}\left(\left\|w_{\ell}\right\|_{\mathcal{C}_{\ell}}\right)
$$

Now, for every $\tilde{w}=\left(\tilde{w}_{1}, \tilde{w}_{2}, \ldots, \tilde{w}_{18}\right), w=\left(w_{1}, w_{2}, \ldots, w_{18}\right) \in \mathcal{O}_{\kappa_{\ell}}$, we have

$$
\begin{aligned}
& \left|\left(\mathcal{T}_{1}^{(\ell)} \tilde{w}+\mathcal{T}_{2}^{(\ell)} w\right)(s)\right| \\
& \leq \int_{0}^{s} \frac{(s-\omega)^{j-1}}{\Gamma(j)}\left|\mathcal{H}_{\ell}\left(\omega, \tilde{w}_{\ell}(\omega), \mathfrak{D}^{k} \tilde{w}_{\ell}(\omega), \tilde{w}_{\ell}^{\prime}(\omega)\right)\right| d \omega \\
& +\left|\eta_{3}\right|\left(\frac{1}{\left|\eta_{1}+\eta_{2}\right|}+\frac{\left|A_{0}\right|+s}{\left|A_{1}\right|}\right) \int_{0}^{1} \int_{0}^{\omega}\left|\mathcal{H}_{\ell}\left(\zeta, w_{\ell}(\zeta), \mathfrak{D}^{k} w_{\ell}(\zeta), w_{\ell}^{\prime}(\zeta)\right)\right| d \zeta d \omega \\
& +\frac{\left|\eta_{2}\right|}{\left|\eta_{1}+\eta_{2}\right|} \int_{0}^{1} \frac{(1-\omega)^{j-1}}{\Gamma(j)}\left|\mathcal{H}_{\ell}\left(\omega, w_{\ell}(\omega), \mathfrak{D}^{k} w_{\ell}(\omega), w_{\ell}^{\prime}(\omega)\right)\right| d \omega \\
& +\frac{\left|A_{0}\right|+s}{\left|A_{1}\right|}\left[\frac{\left|\eta_{2}\right|}{\Gamma(j-2 k)} \int_{0}^{1}(1-\omega)^{j-2 k-1}\left|\mathcal{H}_{\ell}\left(\omega, w_{\ell}(\omega), \mathfrak{D}^{k} w_{\ell}(\omega), w_{\ell}^{\prime}(\omega)\right)\right| d \omega\right. \\
& \left.+\frac{\left|\eta_{1}\right|}{\Gamma(j-k)} \int_{0}^{1}(1-\omega)^{j-k-1}\left|\mathcal{H}_{\ell}\left(\omega, w_{\ell}(\omega), \mathfrak{D}^{k} w_{\ell}(\omega), w_{\ell}^{\prime}(\omega)\right)\right| d \omega\right] \\
& \leq \int_{0}^{s} \frac{(s-\omega)^{j-1}}{\Gamma(j)} \mathcal{W}_{\ell}(\omega) \mathcal{P}_{\ell}\left(\left|\tilde{w}_{\ell}(\omega)\right|+\left|\mathfrak{D}^{k} \tilde{w}_{\ell}(\omega)\right|+\left|\tilde{w}_{\ell}^{\prime}(\omega)\right|\right) d \omega \\
& +\left|\eta_{3}\right|\left(\frac{1}{\left|\eta_{1}+\eta_{2}\right|}+\frac{\left|A_{0}\right|+s}{\left|A_{1}\right|}\right) \\
& \times \int_{0}^{1} \int_{0}^{\omega} \mathcal{W}_{\ell}(\zeta) \mathcal{P}_{\ell}\left(\left|w_{\ell}(\zeta)\right|+\left|\mathfrak{D}^{k} w_{\ell}(\zeta)\right|+\left|w_{\ell}^{\prime}(\zeta)\right|\right) d \zeta d \omega \\
& +\frac{\left|\eta_{2}\right|}{\left|\eta_{1}+\eta_{2}\right|} \int_{0}^{1} \frac{(1-\omega)^{j-1}}{\Gamma(j)} \mathcal{W}_{\ell}(\zeta) \mathcal{P}_{\ell}\left(\left|w_{\ell}(\omega)\right|+\left|\mathfrak{D}^{k} w_{\ell}(\omega)\right|+\left|w_{\ell}^{\prime}(\omega)\right|\right) d \omega \\
& +\frac{\left|A_{0}\right|+s}{\left|A_{1}\right|} \\
& \times\left[\frac{\left|\eta_{2}\right|}{\Gamma(j-2 k)} \int_{0}^{1}(1-\omega)^{j-2 k-1} \mathcal{W}_{\ell}(\zeta) \mathcal{P}_{\ell}\left(\left|w_{\ell}(\omega)\right|+\left|\mathfrak{D}^{k} w_{\ell}(\omega)\right|+\left|w_{\ell}^{\prime}(\omega)\right|\right) d \omega\right. \\
& \left.+\frac{\left|\eta_{1}\right|}{\Gamma(j-k)} \int_{0}^{1}(1-\omega)^{j-k-1} \mathcal{W}_{\ell}(\zeta) \mathcal{P}_{\ell}\left(\left|w_{\ell}(\omega)\right|+\left|\mathfrak{D}^{k} w_{\ell}(\omega)\right|+\left|w_{\ell}^{\prime}(\omega)\right|\right) d \omega\right] \\
& \leq\left\|\mathcal{W}_{\ell}\right\| \tilde{\mathcal{P}}_{\ell}\left[\frac{1}{\Gamma(j+1)}+\left(\frac{1+\left|A_{0}\right|}{\left|A_{1}\right|}\right) \frac{\left|\eta_{1}\right|}{\Gamma(j-k+1)}+\left(\frac{1+\left|A_{0}\right|}{\left|A_{1}\right|}+\frac{1}{\left|\eta_{1}+\eta_{2}\right|}\right) \frac{\left|\eta_{3}\right|}{2}\right. \\
& \left.+\left(\frac{1+\left|A_{0}\right|}{\left|A_{1}\right| \Gamma(j-2 k+1)}+\frac{1}{\left|\eta_{1}+\eta_{2}\right| \Gamma(j+1)}\right)\left|\eta_{2}\right|\right] \\
& =\left\|\mathcal{W}_{\ell}\right\| \tilde{\mathcal{P}}_{\ell} \mathcal{F}_{0}^{*} \text {, }
\end{aligned}
$$


and

$$
\begin{aligned}
&\left|\mathfrak{D}^{k} \mathcal{T}_{1}^{(\ell)} \tilde{w}(s)+\mathfrak{D}^{k} \mathcal{T}_{2}^{(\ell)} w(s)\right| \leq \int_{0}^{s} \frac{(s-\omega)^{j-k-1}}{\Gamma(j-k)}\left|\mathcal{H}_{\ell}\left(\omega, \tilde{w}_{\ell}(\omega), \mathfrak{D}^{k} \tilde{w}_{\ell}(\omega), \tilde{w}_{\ell}^{\prime}(\omega)\right)\right| d \omega \\
& \quad+\frac{\left|\eta_{3}\right| s^{1-k}}{\Gamma(2-k)\left|A_{1}\right|} \int_{0}^{1} \int_{0}^{\omega}\left|\mathcal{H}_{\ell}\left(\zeta, w_{\ell}(\zeta), \mathfrak{D}^{k} w_{\ell}(\zeta), w_{\ell}^{\prime}(\zeta)\right)\right| d \zeta d \omega \\
& \quad+\frac{s^{1-k}}{\Gamma(2-k)\left|A_{1}\right|}\left[\frac{\left|\eta_{2}\right|}{\Gamma(j-2 k)} \int_{0}^{1}(1-\omega)^{j-2 k-1}\left|\mathcal{H}_{\ell}\left(\omega, w_{\ell}(\omega), \mathfrak{D}^{k} w_{\ell}(\omega), w_{\ell}^{\prime}(\omega)\right)\right| d \omega\right. \\
&\left.\quad+\frac{\left|\eta_{1}\right|}{\Gamma(j-k)} \int_{0}^{1}(1-\omega)^{j-k-1}\left|\mathcal{H}_{\ell}\left(\omega, w_{\ell}(\omega), \mathfrak{D}^{k} w_{\ell}(\omega), w_{\ell}^{\prime}(\omega)\right)\right| d \omega\right] \\
& \leq\left\|\mathcal{W}_{\ell}\right\| \tilde{\mathcal{P}}_{\ell}\left[\frac{1}{\Gamma(j-k+1)}+\frac{\left|\eta_{1}\right|}{\Gamma(2-k)\left|A_{1}\right|}\left(\frac{\left|\eta_{3}\right|}{\Gamma(j-k+1)}+\frac{\mid j-2 k+1)}{\Gamma(j-k}\right)\right] \\
&=\left\|\mathcal{W}_{\ell}\right\| \tilde{\mathcal{P}}_{\ell} \mathcal{F}_{1}^{*} \cdot
\end{aligned}
$$

By using similar computations, we have

$$
\begin{aligned}
& \left|\left(\mathcal{T}_{1}^{(\ell)} \tilde{w}\right)^{\prime}(s)+\left(\mathcal{T}_{2}^{(\ell)} w\right)^{\prime}(s)\right| \\
& \leq \int_{0}^{s} \frac{(s-\omega)^{j-2}}{\Gamma(j-1)}\left|\mathcal{H}_{\ell}\left(\omega, \tilde{w}_{\ell}(\omega), \mathfrak{D}^{k} \tilde{w}_{\ell}(\omega), \tilde{w}_{\ell}^{\prime}(\omega)\right)\right| d \omega \\
& +\frac{\left|\eta_{3}\right|}{\left|A_{1}\right|} \int_{0}^{1} \int_{0}^{\omega}\left|\mathcal{H}_{\ell}\left(\zeta, w_{\ell}(\zeta), \mathfrak{D}^{k} w_{\ell}(\zeta), w_{\ell}^{\prime}(\zeta)\right)\right| d \zeta d \omega \\
& +\frac{1}{\left|A_{1}\right|}\left[\frac{\left|\eta_{2}\right|}{\Gamma(j-2 k)} \int_{0}^{1}(1-\omega)^{j-2 k-1}\left|\mathcal{H}_{\ell}\left(\omega, w_{\ell}(\omega), \mathfrak{D}^{k} w_{\ell}(\omega), w_{\ell}^{\prime}(\omega)\right)\right| d \omega\right. \\
& \left.+\frac{\left|\eta_{1}\right|}{\Gamma(j-k)} \int_{0}^{1}(1-\omega)^{j-k-1}\left|\mathcal{H}_{\ell}\left(\omega, w_{\ell}(\omega), \mathfrak{D}^{k} w_{\ell}(\omega), w_{\ell}^{\prime}(\omega)\right)\right| d \omega\right] \\
& \leq\left\|\mathcal{W}_{\ell}\right\| \tilde{\mathcal{P}}_{\ell}\left[\frac{1}{\Gamma(j)}+\frac{1}{\left|A_{1}\right|}\left(\frac{\left|\eta_{1}\right|}{\Gamma(j-k+1)}+\frac{\left|\eta_{2}\right|}{\Gamma(j-2 k+1)}+\frac{\left|\eta_{3}\right|}{2}\right)\right] \\
& =\left\|\mathcal{W}_{\ell}\right\| \tilde{\mathcal{P}}_{\ell} \mathcal{F}_{2}^{*} \text {. }
\end{aligned}
$$

This yields

$$
\left\|\mathcal{T}_{1} \tilde{w}+\mathcal{T}_{2} w\right\|_{\mathcal{C}}=\sum_{\ell=1}^{18}\left\|\mathcal{T}_{1}^{(\ell)} \tilde{w}+\mathcal{T}_{2}^{(k)} w\right\|_{\mathcal{C}_{\ell}} \leq\left\|\mathcal{W}_{\ell}\right\| \tilde{\mathcal{P}}_{\ell}\left(\mathcal{F}_{0}^{*}+\mathcal{F}_{1}^{*}+\mathcal{F}_{2}^{*}\right) \leq \kappa_{\ell}
$$

and so $\mathcal{T}_{1} \tilde{w}+\mathcal{T}_{2} w \in \mathcal{O}_{\kappa_{\ell}}$. Also, the continuity of $\mathcal{H}_{\ell}$ follows from the continuity of the operator $\mathcal{T}_{1}$.

Now, we shall show that $\mathcal{T}_{1}$ is uniformly bounded. As for this purpose, we have

$$
\begin{aligned}
\left|\left(\mathcal{T}_{1}^{(\ell)} w\right)(s)\right| & \leq \int_{0}^{s} \frac{(s-\omega)^{j-1}}{\Gamma(j)}\left|\mathcal{H}_{\ell}\left(\omega, w_{\ell}(\omega), \mathfrak{D}^{k} w_{\ell}(\omega), w_{\ell}^{\prime}(\omega)\right)\right| d \omega \\
& \leq \frac{1}{\Gamma(j+1)}\left\|\mathcal{W}_{\ell}\right\| \mathcal{P}_{\ell}\left(\left|w_{\ell}(\omega)\right|+\left|\mathfrak{D}^{k} w_{\ell}(\omega)\right|+\left|w_{\ell}^{\prime}(\omega)\right|\right)
\end{aligned}
$$


for all $w \in \mathcal{O}_{\kappa_{\ell}}$. Also,

$$
\begin{aligned}
\left|\left(\mathfrak{D}^{k} \mathcal{T}_{1}^{(\ell)} w\right)(s)\right| & \leq \int_{0}^{s} \frac{(s-\omega)^{j-k-1}}{\Gamma(j-k)}\left|\mathcal{H}_{\ell}\left(\omega, w_{\ell}(\omega), \mathfrak{D}^{k} w_{\ell}(\omega), w_{\ell}^{\prime}(\omega)\right)\right| d \omega \\
& \leq \frac{1}{\Gamma(j-k+1)}\left\|\mathcal{W}_{\ell}\right\| \mathcal{P}_{\ell}\left(\left|w_{\ell}(\omega)\right|+\left|\mathfrak{D}^{k} w_{\ell}(\omega)\right|+\left|w_{\ell}^{\prime}(\omega)\right|\right),
\end{aligned}
$$

and

$$
\left|\left(\mathcal{T}_{1}^{(\ell)} w\right)^{\prime}(s)\right| \leq \frac{1}{\Gamma(j)}\left\|\mathcal{W}_{\ell}\right\| \mathcal{P}_{\ell}\left(\left|w_{\ell}(\omega)\right|+\left|\mathfrak{D}^{k} w_{\ell}(\omega)\right|+\left|w_{\ell}^{\prime}(\omega)\right|\right),
$$

for all $w \in \mathcal{O}_{\kappa_{\ell}}$. Thus,

$$
\left\|\mathcal{T}_{1} w\right\|_{\mathcal{C}}=\sum_{\ell=1}^{18}\left\|\mathcal{T}_{1}^{(\ell)} w\right\|_{\mathcal{C}_{\ell}} \leq\left\{\frac{j+1}{\Gamma(j+1)}+\frac{1}{\Gamma(j-k+1)}\right\} \sum_{\ell=1}^{18}\left\|\mathcal{W}_{\ell}\right\| \mathcal{P}_{\ell}\left(\left\|w_{\ell}\right\|_{\mathcal{C}_{\ell}}\right)
$$

which shows that $\mathcal{T}_{1}$ is uniformly bounded on $\mathcal{O}_{\kappa \ell}$.

Now, we shall prove that $\mathcal{T}_{1}$ is compact on $\mathcal{O}_{\kappa_{\ell}}$. For this, let $s_{1}, s_{2} \in[0,1]$ with $s_{1}<s_{2}$. Then, we have

$$
\begin{aligned}
\left|\left(\mathcal{T}_{1}^{(\ell)} w\right)\left(s_{2}\right)-\left(\mathcal{T}_{1}^{(k)} w\right)\left(s_{1}\right)\right| & \\
\leq & \mid \int_{0}^{s_{2}} \frac{\left(s_{2}-\omega\right)^{j-1}}{\Gamma(j)} \mathcal{H}_{\ell}\left(\omega, w_{\ell}(\omega), \mathfrak{D}^{k} w_{\ell}(\omega), w_{\ell}^{\prime}(\omega)\right) d \omega \\
& \quad-\int_{0}^{s_{1}} \frac{\left(s_{1}-\omega\right)^{j-1}}{\Gamma(j)} \mathcal{H}_{\ell}\left(\omega, w_{\ell}(\omega), \mathfrak{D}^{k} w_{\ell}(\omega), w_{\ell}^{\prime}(\omega)\right) d \omega \mid \\
\leq & \left|\int_{0}^{s_{1}} \frac{\left(s_{2}-\omega\right)^{j-1}-\left(s_{1}-\omega\right)^{j-1}}{\Gamma(j)} \mathcal{H}_{\ell}\left(\omega, w_{\ell}(\omega), \mathfrak{D}^{k} w_{\ell}(\omega), w_{\ell}^{\prime}(\omega)\right) d \omega\right| \\
& +\left|\int_{s_{1}}^{s_{2}} \frac{\left(s_{2}-\omega\right)^{j-1}}{\Gamma(j)} \mathcal{H}_{\ell}\left(\omega, w_{\ell}(\omega), \mathfrak{D}^{k} w_{\ell}(\omega), w_{\ell}^{\prime}(\omega)\right) d \omega\right| \\
& +\int_{0}^{s_{1}} \frac{\left(s_{2}-\omega\right)^{j-1}-\left(s_{1}-\omega\right)^{j-1}}{\Gamma(j)}\left|\mathcal{H}_{\ell}\left(\omega, w_{\ell}(\omega), \mathfrak{D}^{k} w_{\ell}(\omega), w_{\ell}^{\prime}(\omega)\right)\right| d \omega \\
\leq & \left\{\frac{s_{2}-s_{1}^{j}-\left(s_{2}-s_{1}\right)^{j}}{\Gamma(j)}+\frac{\left(s_{2}-s_{1}\right)^{j}}{\Gamma(j+1)}\right\}\left\|\mathcal{H}_{\ell}\right\| \mathcal{P}_{\ell}\left(\left\|w_{\ell}\right\|_{\mathcal{C}_{\ell}}\right) .
\end{aligned}
$$

Moreover, $\left|\left(\mathcal{T}_{1}^{(\ell)} w\right)\left(s_{2}\right)-\left(\mathcal{T}_{1}^{(\ell)} w\right)\left(s_{1}\right)\right| \rightarrow 0$ as $s_{1} \rightarrow s_{2}$. Also, we have

$$
\begin{aligned}
& \lim _{s_{1} \rightarrow s_{2}}\left|\left(\mathfrak{D}^{k} \mathcal{T}_{1}^{(\ell)} w\right)\left(s_{2}\right)-\left(\mathfrak{D}^{k} \mathcal{T}_{1}^{(\ell)} w\right)\left(s_{1}\right)\right|=0, \\
& \lim _{s_{1} \rightarrow s_{2}}\left|\left(\mathcal{T}_{1}^{(\ell)} w\right)^{\prime}\left(s_{2}\right)-\left(\mathcal{T}_{1}^{(\ell)} w\right)^{\prime}\left(s_{1}\right)\right|=0 .
\end{aligned}
$$

Hence, $\left\|\left(\mathcal{T}_{1} w\right)\left(s_{2}\right)-\left(\mathcal{T}_{1} w\right)\left(s_{1}\right)\right\|_{\mathcal{C}}$ converges to zero as $s_{1} \rightarrow s_{2}$. Thus, $\mathcal{T}_{1}$ is equicontinuous and so $\mathcal{T}_{1}$ is relatively compact operator on $\mathcal{O}_{\kappa_{\ell}}$. Now, by Arzela-Ascoli theorem, we obtain that $\mathcal{T}_{1}$ is compact on $\mathcal{O}_{\kappa \ell}$. 
Lastly, we need to prove that $\mathcal{T}_{2}$ is a contraction. For this, let $\tilde{w}, w \in \mathcal{O}_{\kappa \ell}$. Thus, we have

$$
\begin{aligned}
&\left|\left(\mathcal{T}_{2}^{(\ell)} \tilde{w}\right)(s)-\left(\mathcal{T}_{2}^{(k)} w\right)(s)\right| \\
& \leq \mid\left|\eta_{3}\right|\left(\frac{1}{\left|\eta_{1}+\eta_{2}\right|}+\frac{\left|A_{0}\right|+s}{\left|A_{1}\right|}\right) \int_{0}^{1} \int_{0}^{\omega} \mathcal{S}_{\ell}(\omega)\left(\left|\tilde{w}_{\ell}(\zeta)-w_{\ell}(\zeta)\right|\right. \\
&\left.+\left|\mathfrak{D}^{k} \tilde{w}_{\ell}(\zeta)-\mathfrak{D}^{k} w_{\ell}(\zeta)\right|+\left|\tilde{w}_{\ell}^{\prime}(\zeta)-w_{\ell}^{\prime}(\zeta)\right|\right) d \zeta d \omega \\
&+\frac{\left|\eta_{2}\right|}{\left|\eta_{1}+\eta_{2}\right|} \int_{0}^{1} \frac{(1-\omega)^{j-1}}{\Gamma(j)} \mathcal{S}_{\ell}(\omega)\left(\left|\tilde{w}_{\ell}(\omega)-w_{\ell}(\omega)\right|+\left|\mathfrak{D}^{k} \tilde{w}_{\ell}(\omega)-\mathfrak{D}^{k} w_{\ell}(\omega)\right|\right. \\
&\left.+\left|\tilde{w}_{\ell}^{\prime}(\omega)-w_{\ell}^{\prime}(\omega)\right|\right) d \omega \\
&+\frac{\left|A_{0}\right|+s}{\left|A_{1}\right|}\left[\frac { | \eta _ { 2 } | } { \Gamma ( j - 2 k ) } \int _ { 0 } ^ { 1 } ( 1 - \omega ) ^ { j - 2 k - 1 } \mathcal { S } _ { \ell } ( \omega ) \left(\left|\tilde{w}_{\ell}(\omega)-w_{\ell}(\omega)\right|\right.\right. \\
&\left.+\left|\mathfrak{D}^{k} \tilde{w}_{\ell}(\omega)-\mathfrak{D}^{k} w_{\ell}(\omega)\right|+\left|\tilde{w}_{\ell}^{\prime}(\omega)-w_{\ell}^{\prime}(\omega)\right|\right) d \omega \\
&+\frac{\left|\eta_{1}\right|}{\Gamma(j-k)} \int_{0}^{1}(1-\omega)^{j-k-1} \mathcal{S}_{\ell}(\omega)\left(\left|\tilde{w}_{\ell}(\omega)-w_{\ell}(\omega)\right|\right. \\
&\left.\left.+\left|\mathfrak{D}^{k} \tilde{w}_{\ell}(\omega)-\mathfrak{D}^{k} w_{\ell}(\omega)\right|+\left|\tilde{w}_{\ell}^{\prime}(\omega)-w_{\ell}^{\prime}(\omega)\right|\right) d \omega\right] \\
& \leq\left\|\mathcal{S}_{\ell}\right\|\left[\frac{1}{\Gamma(j+1)}+\left(\frac{1+\left|A_{0}\right|}{\left|A_{1}\right|}\right) \frac{\left|\eta_{1}\right|}{\Gamma(j-k+1)}\right. \\
&+\left(\frac{1+\left|A_{0}\right|}{\left|A_{1}\right| \Gamma(j-2 k+1)}+\frac{1}{\left|\eta_{1}+\eta_{2}\right| \Gamma(j+1)}\right)\left|\eta_{2}\right| \\
&\left.+\left(\frac{1+\left|A_{0}\right|}{\left|A_{1}\right|}+\frac{1}{\left|\eta_{1}+\eta_{2}\right|}\right) \frac{\left|\eta_{3}\right|}{2}\right]\left\|\tilde{w}_{\ell}-w_{\ell}\right\| \mathcal{C}_{\ell} \\
&= \mathcal{S}_{\ell}\left\|\mathcal{V}_{0}^{*}\right\| \tilde{w}_{\ell}-w_{\ell} \| \mathcal{C}_{\ell}
\end{aligned}
$$

for each $\ell=1,2, \ldots, 18$, where $\mathcal{V}_{0}^{*}$ is given in (3.8). Also, by similar computations, we have

$$
\begin{aligned}
& \sup _{s \in[0,1]}\left|\left(\mathfrak{D}^{k} \mathcal{T}_{2}^{(\ell)} \tilde{w}\right)(s)-\left(\mathfrak{D}^{k} \mathcal{T}_{2}^{(\ell)} w\right)(s)\right| \leq\left\|\mathcal{S}_{\ell}\right\| \mathcal{V}_{1}^{*}\left\|\tilde{w}_{\ell}-w_{\ell}\right\|_{\mathcal{C}_{\ell}}, \\
& \sup _{s \in[0,1]}\left|\left(\mathcal{T}_{2}^{(\ell)} z\right)^{\prime}(s)-\left(\mathcal{T}_{2}^{(\ell)} y\right)^{\prime}(s)\right| \leq\left\|\mathcal{S}_{\ell}\right\| \mathcal{V}_{2}^{*}\left\|z_{k}-w_{\ell}\right\|_{\mathcal{C}_{\ell}},
\end{aligned}
$$

where $\mathcal{V}_{1}^{*}$ and $\mathcal{V}_{2}^{*}$ are given in (3.9) and (3.10), respectively. Thus, we have

$$
\left\|\mathcal{T}_{2} \tilde{w}-\mathcal{T}_{2} w\right\|_{\mathcal{C}}=\sum_{\ell=1}^{18}\left\|\mathcal{T}_{2}^{(\ell)} \tilde{w}-\mathcal{T}_{2}^{(k)} w\right\|_{\mathcal{C}_{\ell}} \leq\left(\mathcal{V}_{0}^{*}+\mathcal{V}_{1}^{*}+\mathcal{V}_{2}^{*}\right) \sum_{\ell=1}^{18}\left\|\mathcal{S}_{\ell}\right\|\left\|\tilde{w}_{k}-w_{\ell}\right\|_{\mathcal{C}_{\ell}}
$$

and so $\left\|\mathcal{T}_{2} \tilde{w}-\mathcal{T}_{2} w\right\|_{\mathcal{C}} \leq \mathcal{F}\|\tilde{w}-w\|_{\mathcal{C}}$. As $\mathcal{F}<1$, which means that $\mathcal{T}_{2}$ is a contraction on $\mathcal{O}_{\kappa_{\ell}}$. As a result of Theorem 2.5, we infer that $\mathcal{T}$ contains a fixed point that is a solution to the fractional boundary value problem (1.6).

To show the significance of our results, we present the following example. 
Example 3.3 Consider the following system of fractional differential equations:

$$
\left\{\begin{array}{l}
\mathfrak{D}^{1.7} w_{1}(s)=\frac{8 e^{s}\left|w_{1}(s)\right|}{20,000\left(1+\left|w_{1}(s)\right|\right)}+0.0004 e^{s}\left|\mathfrak{D}^{0.04} w_{1}(s)\right|+\frac{2 e^{s}\left|\arcsin w_{1}^{\prime}(s)\right|}{5000}, \\
\mathfrak{D}^{1.7} w_{2}(s)=\frac{e^{s}\left|\arctan w_{2}(s)\right|}{5000}+0.0002 e^{s}\left|\sin \left(\mathfrak{D}^{0.04} w_{2}(s)\right)\right|+\frac{30 e^{s}\left|w_{2}^{\prime}(s)\right|}{150,000\left(1+\left|w_{2}^{\prime}(s)\right|\right)}, \\
\mathfrak{D}^{1.7} w_{3}(s)=\frac{s\left|w_{3}(s)\right|}{6000\left(1+\left|w_{3}(s)\right|\right)}+\frac{4 s\left|\mathfrak{D}^{0.04} w_{3}(s)\right|}{24,000}+\frac{12 s\left|\arcsin w_{3}^{\prime}(s)\right|}{72,000}, \\
\mathfrak{D}^{1.7} w_{4}(s)=0.0009 s\left|\sin w_{4}(s)\right|+\frac{180 s\left|\mathfrak{D}^{0.04} w_{4}(s)\right|}{200,000+200,000\left|\mathfrak{D}^{0.04} w_{4}(s)\right|}+\frac{36 s\left|\arcsin w_{4}^{\prime}(s)\right|}{40,000},
\end{array}\right.
$$

with boundary conditions

$$
\left\{\begin{array}{l}
\frac{3}{2} w_{1}(0)+\frac{7}{4} w_{1}(1)=\frac{17}{6} \int_{0}^{1} \mathfrak{D}^{0.7} w_{1}(\omega) d \omega \\
\frac{3}{2} \mathcal{D}^{0.04} w_{1}(1)+\frac{7}{4} \mathcal{D}^{0.08} w_{1}(1)=\frac{17}{6} \int_{0}^{1} \mathfrak{D}^{0.7} w_{1}(\omega) d \omega \\
\frac{3}{2} w_{2}(0)+\frac{7}{4} w_{2}(1)=\frac{17}{6} \int_{0}^{1} \mathfrak{D}^{0.7} w_{2}(\omega) d \omega \\
\frac{3}{2} \mathcal{D}^{0.04} w_{2}(1)+\frac{7}{4} \mathcal{D}^{0.08} w_{2}(1)=\frac{17}{6} \int_{0}^{1} \mathfrak{D}^{0.7} w_{2}(\omega) d \omega \\
\frac{3}{2} w_{3}(0)+\frac{7}{4} w_{3}(1)=\frac{17}{6} \int_{0}^{1} \mathfrak{D}^{0.7} w_{3}(\omega) d \omega \\
\frac{3}{2} \mathcal{D}^{0.04} w_{3}(1)+\frac{7}{4} \mathcal{D}^{0.08} w_{3}(1)=\frac{17}{6} \int_{0}^{1} \mathfrak{D}^{0.7} w_{3}(\omega) d \omega \\
\frac{3}{2} w_{4}(0)+\frac{7}{4} w_{4}(1)=\frac{17}{6} \int_{0}^{1} \mathfrak{D}^{0.7} w_{4}(\omega) d \omega \\
\frac{3}{2} \mathcal{D}^{0.04} w_{4}(1)+\frac{7}{4} \mathcal{D}^{0.08} w_{4}(1)=\frac{17}{6} \int_{0}^{1} \mathfrak{D}^{0.7} w_{4}(\omega) d \omega
\end{array}\right.
$$

where $j=1.7, k=0.04, \eta_{1}=\frac{3}{2}, \eta_{2}=\frac{7}{4}, \eta_{3}=\frac{17}{6}$, and $\mathfrak{D}^{j}, \mathfrak{D}^{k}$ serve as the Caputo fractional derivative of orders $j$ and $k$, respectively.

Let $\mathcal{H}_{1}, \mathcal{H}_{2}, \mathcal{H}_{3}, \mathcal{H}_{4}:[0,1] \times \mathbb{R} \times \mathbb{R} \times \mathbb{R} \rightarrow \mathbb{R}$ are continuous functions defined by

$$
\left\{\begin{aligned}
\mathcal{H}_{1}(s, w(s), \tilde{w}(s), \tilde{\tilde{w}}(s))= & \frac{8 e^{s}|w(s)|}{20,00(1+|w(s)|)}+0.0004 e^{s}\left|\mathfrak{D}^{0.04} \tilde{w}(s)\right| \\
& +\frac{2 e^{s}|\arcsin \tilde{\tilde{w}}(s)|}{5000}, \\
\mathcal{H}_{2}(s, w(s), \tilde{w}(s), \tilde{\tilde{w}}(s))= & \frac{e^{s}|\arctan w(s)|}{5000}+0.0002 e^{s}\left|\sin \left(\mathfrak{D}^{0.04} \tilde{w}(s)\right)\right| \\
& +\frac{30 e^{s}|\tilde{\tilde{w}}(s)|}{150,000(1+|\tilde{\tilde{w}}(s)|)} \\
\mathcal{H}_{3}(s, w(s), \tilde{w}(s), \tilde{\tilde{w}}(s))= & \frac{s\left|w_{3}(s)\right|}{6000\left(1+\left|w_{3}(s)\right|\right)}+\frac{4 s\left|\mathfrak{D}^{0.04} w_{3}(s)\right|}{24,000} \\
& +\frac{12 s\left|\arcsin w_{3}^{\prime}(s)\right|}{72,000}, \\
\mathcal{H}_{4}(s, w(s), \tilde{w}(s), \tilde{\tilde{w}}(s))= & 0.0009 s|\sin w(s)|+\frac{180 s\left|\mathfrak{D}^{0.04} \tilde{w}(s)\right|}{200,000+200,000\left|\mathfrak{D}^{0.04} \tilde{w}(s)\right|} \\
& +\frac{36 s|\arcsin \tilde{\tilde{w}}(s)|}{40,000} .
\end{aligned}\right.
$$

Let $w_{1}, w_{2}, \tilde{w}_{1}, \tilde{w}_{2}, \tilde{\tilde{w}}_{1}, \tilde{\tilde{w}}_{2} \in \mathbb{R}$. Then, we have

$$
\begin{aligned}
& \left|\mathcal{H}_{1}\left(s, w_{1}(s), \tilde{w}_{1}(s), \tilde{\tilde{w}}_{1}(s)\right)-\mathcal{H}_{1}\left(s, w_{2}(s), \tilde{w}_{2}(s), \tilde{\tilde{w}}_{2}(s)\right)\right| \\
& \quad \leq \frac{e^{s}}{2500}\left(\left|w_{1}(s)-w_{2}(s)\right|+\left|\tilde{w}_{1}(s)-\tilde{w}_{2}(s)\right|+\left|\arcsin \tilde{\tilde{w}}_{1}(s)-\arcsin \tilde{\tilde{w}}_{2}(s)\right|\right) \\
& \quad\left|\mathcal{H}_{2}\left(s, w_{1}(s), \tilde{w}_{1}(s), \tilde{\tilde{w}}_{1}(s)\right)-\mathcal{H}_{2}\left(s, w_{2}(s), \tilde{w}_{2}(s), \tilde{\tilde{w}}_{2}(s)\right)\right| \\
& \quad \leq \frac{e^{s}}{5000}\left(\left|\arctan w_{1}(s)-\arctan w_{2}(s)\right|+\left|\sin \tilde{w}_{1}(s)-\sin \tilde{w}_{2}(s)\right|+\left|\tilde{\tilde{w}}_{1}(s)-\tilde{\tilde{w}}_{2}(s)\right|\right), \\
& \quad \leq \frac{s}{6000}\left(\left|\mathcal{H}_{3}\left(s, w_{1}(s), \tilde{w}_{1}(s), \tilde{\tilde{w}}_{1}(s)\right)-\mathcal{H}_{3}\left(s, w_{2}(s), \tilde{w}_{2}(s), \tilde{\tilde{w}}_{2}(s)\right)\right|+\left|\tilde{w}_{1}(s)-\tilde{w}_{2}(s)\right|+\left|\arcsin \tilde{\tilde{w}}_{1}(s)-\arcsin \tilde{\tilde{w}}_{2}(s)\right|\right),
\end{aligned}
$$




$$
\begin{aligned}
& \left|\mathcal{H}_{4}\left(s, w_{1}(s), \tilde{w}_{1}(s), \tilde{\tilde{w}}_{1}(s)\right)-\mathcal{H}_{4}\left(s, w_{2}(s), \tilde{w}_{2}(s), \tilde{\tilde{w}}_{2}(s)\right)\right| \\
& \quad \leq \frac{9 s}{10,000}\left(\left|\sin w_{1}(s)-\sin w_{2}(s)\right|+\left|\tilde{w}_{1}(s)-\tilde{w}_{2}(s)\right|+\left|\arcsin \tilde{\tilde{w}}_{1}(s)-\arcsin \tilde{\tilde{w}}_{2}(s)\right|\right) .
\end{aligned}
$$

Here,

$$
\begin{array}{llll}
\mathcal{S}_{1}(s)=\frac{e^{s}}{2500}, & \mathcal{S}_{2}(s)=\frac{e^{s}}{5000}, & \mathcal{S}_{3}(s)=\frac{s}{6000}, & \mathcal{S}_{4}(s)=\frac{9 s}{10,000}, \\
\left\|\mathcal{S}_{1}\right\|=\frac{1}{2500}, & \left\|\mathcal{S}_{2}\right\|=\frac{1}{5000}, & \left\|\mathcal{S}_{3}\right\|=\frac{1}{6000}, & \left\|\mathcal{S}_{4}\right\|=\frac{9}{10,000} .
\end{array}
$$

Let $\mathcal{P}_{1}, \mathcal{P}_{2}, \mathcal{P}_{3}, \mathcal{P}_{4}:[0, \infty) \rightarrow \mathbb{R}$ be identity functions. Then, we obtain

$$
\begin{aligned}
\left|\mathcal{H}_{1}\left(s, w(s), \mathfrak{D}^{0.04} w(s), w^{\prime}(s)\right)\right| & \leq \frac{e^{s}}{2500}\left(|w|+|\mathfrak{D} w|+\left|\arcsin w^{\prime}\right|\right) \\
& \leq \frac{e^{s}}{2500}\left(|w|+|\mathfrak{D} w|+\left|w^{\prime}\right|\right), \\
\left|\mathcal{H}_{2}\left(s, w(s), \mathfrak{D}^{0.04} w(s), w^{\prime}(s)\right)\right| & \leq \frac{e^{s}}{5000}\left(|\arctan w|+|\sin (\mathfrak{D} w)|+\left|w^{\prime}\right|\right) \\
& \leq \frac{e^{s}}{5000}\left(|w|+|\mathfrak{D} w|+\left|w^{\prime}\right|\right), \\
\left|\mathcal{H}_{3}\left(s, w(s), \mathfrak{D}^{0.04} w(s), w^{\prime}(s)\right)\right| & \leq \frac{s}{6000}\left(|w|+|\mathfrak{D} w|+\left|\arcsin w^{\prime}\right|\right) \\
& \leq \frac{s}{6000}\left(|w|+|\mathfrak{D} w|+\left|w^{\prime}\right|\right), \\
\left|\mathcal{H}_{4}\left(s, w(s), \mathfrak{D}^{0.04} w(s), w^{\prime}(s)\right)\right| & \leq \frac{9 s}{10,000}\left(|\sin w|+|\mathfrak{D} w|+\left|\arcsin w^{\prime}\right|\right) \\
& \leq \frac{9 s}{10,000}\left(|w|+|\mathfrak{D} w|+\left|w^{\prime}\right|\right),
\end{aligned}
$$

where the continuous function $\mathcal{W}_{1}, \mathcal{W}_{2}, \mathcal{W}_{3}, \mathcal{W}_{4}:[0,1] \rightarrow \mathbb{R}$ are defined by

$$
\mathcal{W}_{1}(s)=\frac{e^{s}}{2500}, \quad \mathcal{W}_{2}(s)=\frac{e^{s}}{5000}, \quad \mathcal{W}_{3}(s)=\frac{s}{6000}, \quad \mathcal{W}_{4}(s)=\frac{9 s}{10,000}
$$

Furthermore, $\mathcal{V}_{0}^{*} \simeq 5.642, \mathcal{V}_{1}^{*} \simeq 4.084$, and $\mathcal{V}_{2}^{*} \simeq 4.019$, thus

$$
\mathcal{F}:=\left(\mathcal{V}_{0}^{*}+\mathcal{V}_{1}^{*}+\mathcal{V}_{2}^{*}\right)\left(\left\|\mathcal{S}_{1}\right\|+\left\|\mathcal{S}_{2}\right\|+\left\|\mathcal{S}_{3}\right\|+\left\|\mathcal{S}_{4}\right\|\right) \simeq 0.023<1
$$

Hence by Theorem 3.2, the proposed problem (3.13)-(3.14) has a solution.

\section{Conclusion}

The chemical graph theory is a versatile subject that uses practical and theoretical methods to study the molecular structure of a chemical substance as a graph, with specific mathematical challenges in consideration. In recent decades, the rapid development of this field has given us numerous groundbreaking and distinctive ideas and methods for such research. In this article, we used the framework of a cyclohexane graph to illustrate the fractional boundary value problem in the sense of the Caputo fractional derivative. The Schaefer and Krasnoselskii fixed point theorems were used to examine the existence 
of solutions to the suggested problem. Our method is easy to implement and may be used on a wide range of graphs, including chordal bipartite graphs, which have many applications in computer networking and biology.

\section{Acknowledgements}

The authors would like to thank the anonymous referees for their valuable comments and suggestions, which helped to improve the paper.

\section{Funding}

The research of JJN has been partially supported by the Agencia Estatal de Investigacion (AEI) of Spain, co-financed by the European Fund for Regional Development (FEDER), project MTM2016-75140-P; and by Xunta de Galicia under grant ED431C 2019/02

Availability of data and materials

Not applicable.

\section{Declarations}

\section{Competing interests}

The authors declare that they have no competing interests.

\section{Authors' contributions}

All authors contributed equally to the manuscript and typed, read, and approved the final manuscript.

\section{Author details}

${ }^{1}$ School of Science, Nanjing University of Science and Technology, Nanjing 210094, China. ${ }^{2}$ Department of Mathematics and Statistics, Faculty of Science and Technology, Thammasat University Rangsit Center, Pathum Thani 12120, Thailand. ${ }^{3}$ Instituto de Matemáticas, Universidade de Santiago de Compostela, 15782 Santiago de Compostela, Spain.

\section{Publisher's Note}

Springer Nature remains neutral with regard to jurisdictional claims in published maps and institutional affiliations.

Received: 28 June 2021 Accepted: 22 December 2021 Published online: 04 January 2022

\section{References}

1. Lakshmikantham, V., Leela, S., Devi, S.V.: Theory of Fractional Dynamic Systems. Cambridge Academic, Cambridge (2009)

2. Miller, K.S., Ross, B.: An Introduction to the Fractional Calculus and Fractional Differential Equation. Wiley, New York (1993)

3. Oldham, K.B., Spanier, J.: The Fractional Calculus. Academic Press, New York (1974)

4. Podlubny, I.: Fractional Differential Equations. Academic Press, New York (1999)

5. Machado, J.A.T., Kiryakova, V., Mainardi, F.: Recent history of fractional calculus. Commun. Nonlinear Sci. Numer. Simul. 16, 1140-1153 (2011). https://doi.org/10.1016/j.cnsns.2010.05.027

6. Baleanu, D., Diethelm, K., Scalas, E., Trujillo, J.J.: Fractional Calculus Models and Numerical Methods. Series on Complexity, Nonlinearity and Chaos. World Scientific, London (2012)

7. Qiu, T., Bai, Z.: Existence of positive solution for singular fractional equations. Electron. J. Differ. Equ. $2008,146(2008)$

8. Sabatier, J., Agarwal, O.P., Machado, J.A.T.: Advances in Fractional Calculus, Theoretical Developments and Applications in Physics and Engineering. Springer, Berlin (2007)

9. Agarwal, R.P., Lakshmikantham, V., Nieto, J.J.: On the concept of solution for fractional differential equations with uncertainty. Nonlinear Anal., Theory Methods Appl. 72, 2859-2862 (2010). https://doi.org/10.1016/j.na.2009.11.029

10. Oz, D., Karaca, I.: Positive solutions of m-point fractional boundary value problem on the half line. Fract. Differ. Calc. 9(2), 209-226 (2019). https://doi.org/10.7153/fdc-2019-09-14

11. Wang, F., Liu, L., Wu, Y.: Existence and uniqueness of solutions for a class of higher-order fractional boundary value problems with the nonlinear term satisfying some inequalities. J. Inequal. Appl. 196 (2020). https://doi.org/10.1186/s13660-020-02463-0

12. Karaca, I.Y., Oz, D.: Existence of solutions for a fractional-order boundary-value problem. Ukr. Math. J. 72, 1907-1920 (2021). https://doi.org/10.1007/s11253-021-01897-z

13. Karaca, I.Y., Oz, D.: Positive solutions for fractional-order nonlinear boundary value problems on infinite interval. Int. J. Nonlinear Anal. Appl. 12(1), 317-335 (2021). https://doi.org/10.22075/IJNAA.2021.4800

14. Turab, A., Sintunavarat, W.: A unique solution of the iterative boundary value problem for a second-order differential equation approached by fixed point results. Alex. Eng. J. 60(6), 5797-5802 (2021). https://doi.org/10.1016/j.aej.2021.04.031

15. Zhang, S.: The existence of a positive solution for nonlinear fractional differential equation. J. Math. Anal. Appl. 252, 804-812 (2000). https://doi.org/10.1006/jmaa.2000.7123

16. Zhang, S.: Existence of positive solutions for some class of nonlinear fractional equation. J. Math. Anal. Appl. 278, 136-148 (2003). https://doi.org/10.1016/S0022-247X(02)00583-8 
17. Hashim, I., Abdulaziz, O., Momani, S.: Homotopy analysis method for fractional IVPs. Commun. Nonlinear Sci. Numer. Simul. 14, 674-684 (2009). https://doi.org/10.1016/j.cnsns.2007.09.014

18. Al-Mdallal, M., Syam, M.I., Anwar, M.N.: A collocation-shooting method for solving fractional boundary value problems. Commun. Nonlinear Sci. Numer. Simul. 15, 3814-3822 (2010). https://doi.org/10.1016/j.cnsns.2010.01.020

19. Oz, D., Karaca, I.Y.: Positive solutions for $m$-point $p$-Laplacian fractional boundary value problem involving Riemann-Liouville fractional integral boundary conditions on the half line. Filomat 34(9), 3161-3173 (2020). https://doi.org/10.2298/FIL20091610

20. Lumer, G.: Connecting of local operators and evolution equations on a network. Lect. Notes Math. 787, 219-234 (1985). https://doi.org/10.1007/BFb0086338

21. Zavgorodnii, M.G., Pokornyi, Y.V.: On the spectrum of second-order boundary value problems on spatial networks. Usp. Mat. Nauk 44, 220-221 (1989)

22. Gordeziani, D.G., Kupreishvli, M., Meladze, H.V., Davitashvili, T.D.: On the solution of boundary value problem for differential equations given in graphs. Appl. Math. Lett. 13, 80-91 (2008)

23. Graef, J.R., Kong, L.J., Wang, M.: Existence and uniqueness of solutions for a fractional boundary value problem on a graph. Fract. Calc. Appl. Anal. 17, 499-510 (2014). https://doi.org/10.2478/s13540-014-0182-4

24. Mehandiratta, V., Mehra, M., Leugering, G.: Existence and uniqueness results for a nonlinear Caputo fractional boundary value problem on a star graph. J. Math. Anal. Appl. 477(2), 1243-1264 (2019). https://doi.org/10.1016/j.jmaa.2019.05.011

25. Mophou, G., Leugering, G., Fotsing, P.S.: Optimal control of a fractional Sturm-Liouville problem on a star graph Optimization 70(3), 659-687 (2021). https://doi.org/10.1080/02331934.2020.1730371

26. Etemad, S., Rezapour, S.: On the existence of solutions for fractional boundary value problems on the ethane graph. Adv. Differ. Equ. 2020, 276 (2020). https://doi.org/10.1186/s13662-020-02736-4

27. Baleanu, D., Etemad, S., Mohammadi, H., Rezapour, S.: A novel modeling of boundary value problems on the glucose graph. Commun. Nonlinear Sci. Numer. Simul. 100 (2021). https://doi.org/10.1016/j.cnsns.2021.105844

28. Turab, A., Sintunavarat, W.: The novel existence results of solutions for a nonlinear fractional boundary value problem on the ethane graph. Alex. Eng. J. 60(6), 5365-5374 (2021). https://doi.org/10.1016/.jaej.2021.04.020

29. Zhang, W., Liu, W.: Existence and Ulam's type stability results for a class of fractional boundary value problems on a star graph. Math. Methods Appl. Sci. 43(15), 8568-8594 (2020). https://doi.org/10.1002/mma.6516

30. Smart, D.R.: Fixed Point Theorems. Cambridge University Press, Cambridge (1990)

\section{Submit your manuscript to a SpringerOpen ${ }^{\circ}$ journal and benefit from:}

- Convenient online submission

- Rigorous peer review

- Open access: articles freely available online

- High visibility within the field

- Retaining the copyright to your article

Submit your next manuscript at $\gg$ springeropen.com 